\title{
Articulos
}

\section{¿Cómo saber qué políticas públicas generan mayor bienestar? Aplicación en el sector salud de un modelo para la evaluación de políticas públicas en Nicaragua}

\section{Octavio Martinez Baltodano* y María Haydée Fonseca Mairena}

Recibido: noviembre de 2012 / Aceptado: abril de 2013

El estudio presenta paso a paso la aplicación en el sector salud del modelo CerdaTorche (2005), una metodología para la evaluación de políticas públicas a través de la valoración de los beneficios sociales derivados de la reducción en las tasas de mortalidad. Dicha metodología puede ser aplicada a cualquier sector para evaluar el impacto social de la ejecución de políticas, programas o proyectos y de esta forma reorientar eficazmente los recursos económicos del gobierno. El valor económico de la vida se determina a través de un modelo intertemporal en el que los agentes maximizan sus patrones de consumo y ocio sujetos a una restricción presupuestaria en la que se puede acumular activos a lo largo del ciclo de vida, en ausencia de mercados completos y enfrentando probabilidades exógenas de supervivencia. Este modelo se calibra con datos de la economía nicaragüense durante 2008 y simulaciones a través de modificaciones paramétricas que permiten determinar los beneficios sociales provocados por la reducción de las tasas de mortalidad de las primeras seis causas de muerte en Nicaragua en 2008.

Palabras clave: políticas públicas / sector salud / beneficios sociales / reducción de las tasas de mortalidad

* Estudios y Análisis Económicos de la Secretaría de Políticas Nacionales (SPPN). Managua, Nicaragua. Correo electrónico: omartinez@sppn.gob.ni

**Departamento de Planificación Municipal del Instituto Nicaragüense de Fomento Municipal (INIFOM). Managua, Nicaragua.Correo electrónico:mhfonmai@hotmail.com; maria.fonseca@inifom.gob.ni 


\section{Introducción}

En países como Nicaragua un factor esencial para lograr un mayor desarrollo económico es el mejoramiento en las condiciones y niveles de vida, interviniendo con políticas, programas y proyectos en salud, educación, demografía, pobreza y desigualdad. Sin embargo, los recursos que se pueden invertir son escasos. Por lo tanto, es necesario realizar evaluaciones de las diferentes alternativas entre diferentes sectores y escoger la que maximice los retornos económicos-sociales obtenidos, es decir el bienestar social.

En el cuerpo del presente documento el lector encontrará una propuesta de instrumento económico para la evaluación de políticas, programas y proyectos. Hemos elegido el sector salud para la aplicación y explicación del modelo por ser uno de los sectores más sensibles para el impulso del desarrollo económico.

Con dicha aplicación en el sector salud, además de lograr la presentación y apropiación del modelo económico, la investigación revela resultados interesantes sobre el valor del ocio, las probabilidades de sobrevivencia/muerte, el valor económico de la vida por edad, nivel educativo y sexo y finalmente, el beneficio social de programas de salud que disminuyan la probabilidad de muerte.

El modelo económico propuesto es una aplicación para Nicaragua del modelo Cerda-Torche (2005), una metodología no tradicional para la evaluación de políticas públicas, a través de la valoración de los beneficios sociales derivados de la reducción en las tasas de mortalidad. El objetivo principal del estudio es que el lector se apropie de dicho modelo o al menos despierte su interés en el mismo y pueda, así como lo hemos hecho para el sector salud, implementarlo en otros sectores. De esta forma pretendemos contribuir al desarrollo del acervo de capital humano de nuestro país, datándoles de una herramienta importante para que estemos cada vez más capacitados para el aseguramiento del uso eficaz de los recursos utilizados en inversiones de interés público.

Hoy en día, la evaluación costo-efecto que utiliza como medida los años de vida ajustados por discapacidad (AVAD) se ha convertido en un método aceptado para evaluar políticas públicas en el sector salud. En dicho método se calculan los años de vida saludables perdidos provocados por alguna enfermedad específica y se establece cómo cambian estas condiciones luego de la implementación de la política evaluada. La diferencia entre los efectos de la enfermedad antes y después del proyecto o programa se comparan con los costes necesarios para la implementación del mismo y de esa manera se obtiene un indicador de coste-efecto. Sin embargo, bajo este método sólo es posible comparar alternativas dentro del sector salud y no entre sectores.

Sin embargo, Cerda y Torche (2005) desarrollaron un modelo para la evaluación de políticas públicas en países en vías de desarrollo a través de la valoración de los beneficios sociales derivados de la reducción en las tasas de mortalidad. En el presente documento aplicamos dicha metodología para la evaluación de políticas en el sector salud, esperando facilite su futura implementación en otros sectores y de esta forma contribuya a mejorar la toma de decisiones orientadas a obtener mayores beneficios sociales. 
En el estudio se muestra un modelo de optimización intertemporal con una función de utilidad que depende del consumo de bienes y servicios y del tiempo que se utiliza en ocio. El modelo incorpora un proceso de acumulación de activos y permite obtener la senda óptima de un consumidor que enfrenta probabilidades exógenas de supervivencia.

La metodología implementada es de corte transversal y enfoque retrospectivo, y está basada en análisis cuantitativo con datos de 2008.

El modelo se calibra con las características de ingreso y utilización del tiempo de los nicaragüenses en 2008. Utilizando la encuesta de empleo 2008, se identifican las condiciones de consumidores particulares diferenciados por características socioeconómicas. Las condiciones de supervivencia se calculan a partir de información suministrada por la oficina de estadísticas del MINSA-Central. Estas condiciones de supervivencia surgen de las tablas de mortalidad elaboradas en base a definiciones de cohortes análogos a las de los agentes considerados. Estas curvas son modificadas paramétricamente para hacer simulaciones de los efectos de políticas públicas en las condiciones de mortalidad que afectan a los individuos.

El resultado puede ser interpretado como el beneficio social de un proyecto o de un programa, ya que el carácter optimizante del modelo entrega una medida adecuada del impacto en el bienestar de la población por parte de dichas políticas.

\section{Revisión de literatura}

En 1982, Marin y Psacharopoulos, en su obra "The Reward for Risk in the Labor Market: Evidence from the United Kingdom and a Reconciliation with Other Studies", estimaron la función de precios hedónicos para determinar el valor de la vida. En la función utilizada, el salario anual depende de: años de escolaridad; años de experiencia; el número de semanas trabajadas durante el año; el riesgo del puesto de trabajo; una variable sindical, aproximada por el porcentaje de trabajadores en el sector con convenio colectivo; y el atractivo del puesto de trabajo.

Para calcular la variable de riesgo, Marin y Psacharopoulos (1982) utilizaron dos vías: 1) Tasa de mortalidad general del grupo según edad y clase social a la que pertenecía la persona, comparada con la tasa de mortalidad de cada ocupación y 2) Comparar la tasa de mortalidad general debida a accidentes laborales con la de la ocupación específica. Finalmente, la variable que se incluyó al modelo fue la establecida mediante el segundo método. Los resultados del estudio mostraron que el valor de la vida era de 2,245,000 libras esterlinas de 1982 para los trabajadores no manuales y 686,000 para los trabajadores manuales.

Por su parte, Riera, Ripoll y Mateu (2007) utilizan también el método de precios hedónicos en "Estimación del valor estadístico de la vida en España: Una aplicación del Método de Salarios Hedónicos”. El estudio se realizó a partir de una amplia base de datos del mercado de trabajo español y de la construcción de índices de riesgo laboral que incorporan simultáneamente la ocupación y la rama de actividad para el período 1997-2000. Los principales resultados de la aplicación constatan una relación positiva entre el riesgo de accidentalidad y el salario percibido y sitúan el valor estadístico de la vida en España en el rango de 2 a 2,7 millones de euros, a precios del 2000. 
Así mismo, Jones-Lee, Hammerton \& Phillips (1985) aplicaron el método de valoración contingente. Para el levantamiento de la información utilizaron una encuesta con 37 preguntas, la cual aplicaron a 1,103 personas. Dichas preguntas se encuentran agrupadas en tres categorías:

1. Características socioeconómicas de la familia

2. Preguntas de percepción-consciencia

3. Preguntas de valoración

Las preguntas de valoración están elaboradas para obtener las tasas marginales de sustitución entre riesgo y dinero. Las preguntas relativas a la valoración del riesgo iniciaban con formato abierto pero se cerraban en la medida en que el entrevistado no tuviera la capacidad de contestar.

Los resultados de este estudio mostraron que el valor de la vida rondaba alrededor de dos millones de libras esterlinas de 1985.

Por otra parte, en 2007, Martínez, Abellán y Pinto elaboraron el estudio titulado "El valor monetario de la vida estadística en España a través de las preferencias declaradas”. En dicho estudio se estima el valor monetario de una vida estadística en España a partir de preferencias declaradas por la población general, esto en el contexto de accidentes de tráfico. La metodología empleada es la de valoración contingente. En la encuesta realizada se intentaron comunicar correctamente los riesgos mediante el recurso de ayuda visual (mostrar imágenes con las consecuencias de accidentes de tráfico). Los valores obtenidos (no inferiores a 2,7 millones de euros corrientes) son similares a otras estimaciones efectuadas en Europa recientemente, si bien un aspecto de la consistencia de estos resultados (cuasi-proporcionalidad) sólo se verifica parcialmente.

Así mismo, en 1989 Viscusi y Moore aplicaron el método de valoración de la vida y las tasas de descuento. En dicho estudio, para el método indirecto (con información secundaria) utilizaron un proceso Markoviano para la elección de la tasa de riesgo de un trabajo durante toda su vida. Entre los principales resultados de la investigación se encuentra que la tasa de descuento utilizada implícitamente por una persona "normal" (con 12 años de educación formal) es de 3,2\% en términos reales. Por otra parte, para una persona con ocho años de escolaridad la tasa es de $6,2 \%$ y finalmente los universitarios muestran tasas de descuento negativas de $-3.3 \%$.

Por otra parte, el método de valoración de la vida y las tasas de descuento con el método directo (con información primaria) fue implementado en 1992 por Cropper, Aydede y Portney. Para ello aplicaron una encuesta a un total de 3,200 personas. Los resultados muestran un grado de variación considerable en dependencia del horizonte temporal contemplado: desde $16,8 \%$ para un horizonte de cinco años hasta 3.8\% para uno de 100. Es importante resaltar que, según el estudio, los encuestados muestran una clara preferencia por el presente.

Por su parte, Murphy y Topel (2006), en su estudio "The Value of Health and Longevity" calculan el valor social de: aumentar las tasas de longevidad de veinte países; el progreso contra un conjunto de enfermedades después de 1970; y el progreso potencial futuro contra las enfermedades. Los resultados muestran que las ganancias acumuladas en la esperanza de vida después de 1900 para un individuo norteamericano representativo fueron de más de \$1.2 millones de dólares del 2000, mientras que después de 1970 las ganancias agregadas sumaron $\$ 3.2$ billones 
por año del PIB. Además los beneficios potenciales de mejoras en la salud futura también son grandes. Por ejemplo, una reducción del $1 \%$ en la mortalidad por cáncer genera un valor de $\$ 500$ mil millones de dólares. Para ello, el estudio se basa en el análisis de la esperanza de vida de Arthur (1981) y Rosen (1988), suponiendo que la disposición a pagar para la salud está determinada por la maximización de la utilidad de toda la vida.

Finalmente, Cerda y Torche (2005) en "El valor económico de reducir tasas de mortalidad: El caso de Chile", presentan una metodología para valorar reducciones de tasas de mortalidad. Empleando el método de capital humano encontraron que los beneficios económicos sociales de erradicar cuatro enfermedades durante los años noventa para la economía chilena fueron: para la neumonía 2.89\% del PIB; para el infarto 3.34\% del PIB; para los accidentes vasculares encefálicos $1.48 \%$ del PIB; y para los tumores malignos al estómago $2.01 \%$ del PIB.

\section{Marco teórico}

\subsection{Medición del valor económico de la vida}

En un contexto de recursos económicos escasos, ¿cómo decidir si invertir en un programa de vacunación contra el neumococo - principal causante de la neumonía en niños y adultos mayores - o invertir en tecnología para un mejor tratamiento y prevención de la insuficiencia renal crónica? Ambos aumentan la esperanza de vida de las personas y la vida humana es invaluable. Para resolver esta disyuntiva - aunque según nuestros preceptos sociales la vida humana tiene un valor infinito resulta necesario utilizar métodos económicos que ayuden a evaluar la pertinencia y eficacia de las políticas, programas y proyectos sociales, para la toma de decisiones. La metodología aquí implementada consiste en identificar qué causa mayores beneficios sociales, multiplicando el valor económico de los individuos por la probabilidad de salvar una vida adicional con la intervención pública. Para valorar económicamente los cambios en las tasas de mortalidad, existen al menos tres métodos que son presentados a continuación.

\subsubsection{Salarios hedónicos}

Se plantea la función de salarios hedónicos del tipo:

$$
\mathrm{W}_{\mathrm{h}}=\mathrm{W}\left(\mathrm{E}_{\mathrm{h}}, \mathrm{R}_{\mathrm{h}}, \mathrm{S}_{\mathrm{h}}, \mathrm{X}_{\mathrm{h}}\right)
$$

En donde el salario de un determinado puesto de trabajo h está en dependencia principalmente de los vectores de características de: capital humano Eh, riesgo Rh, sindicales Sh y medioambientales Xh. (Viscusi, 1993).

El cálculo del valor de la vida mediante este método se obtiene con la derivada parcial del $\mathrm{W}$ respecto al riesgo de muerte, calculando así la disposición marginal a pagar por aceptar un mayor riesgo de muerte. Esto formalmente se conoce como el "valor de una vida estadística".

Sin embargo, dado un desfase temporal, con dicho método resulta estadísticamente imposible separar todas las influencias exógenas. Esto se debe a que 
el riesgo de muerte está en dependencia de cada ocupación profesional (Marin \& Psacharopoulos, 1982), cuya información, en Nicaragua como en el resto de países con similares características de sub desarrollo, es restringida y poco fundamentada, ya que surge de acuerdos entre el sector privado y el gremio sindical (Cropper \& Freeman, 1991). Así mismo, el método no segmenta por sexo ni grupo etario.

\subsubsection{Método indirecto}

En este método se infiere la tasa de descuento implícita utilizando la función de precios hedónicos como restricción en la optimización de cada individuo. Por tanto, cada persona debe decidir entre la probabilidad de muerte y el salario para aceptar dicho riesgo, maximizando sus beneficios (Viscusi \& Moore, 1989).

\subsubsection{Método directo}

Con este método cada individuo debe elegir entre las probabilidades de muerte en el presente y en el futuro. La información es obtenida a través de encuestas dirigidas directamente al grupo de análisis.

\section{Valoración contingente}

Es un método directo que consiste en preguntar al grupo de análisis, el valor presente que le da a un cambio en su bienestar derivado de un cambio en sus probabilidades de morir.

Mientras, el método directo de tasas de descuento cuestiona sobre el valor presente de una determinada posibilidad de morir ahora y en el futuro (Jones-Lee et al., 1985).

Por razones obvias la principal limitante para la aplicación de todo método directo es la necesaria implementación de muchos recursos económicos, humanos y tiempo.

\section{El método del capital humano}

El método del capital humano se basa en la producción. Concretamente se relaciona con los niveles de producción neta de cada individuo y por ende los beneficios de la sociedad si ese individuo sigue viviendo. De esta forma se estima el valor restando la producción del individuo por el resto de su vida menos su consumo. Así:

$$
q=\sum_{t=n}^{T} P_{t n}\left(W_{t}-C_{t}\right)(1+\delta)^{-(t-n)}
$$


En donde:

$\mathrm{Q}=$ Costo por la muerte del individuo a la edad $\mathrm{n}$.

$\mathrm{P}_{\mathrm{tn}}=$ Probabilidad en el año $\mathrm{n}$ de que el individuo siga vivo en el año $t$.

$\mathrm{W}_{\mathrm{t}}=$ Salario en el año $\mathrm{t}$.

$\mathrm{C}_{\mathrm{t}}=$ Consumo personal en el año $\mathrm{t}$.

$\delta=$ Tasa social de descuento

Sin embargo, este modelo presenta dos serias limitantes:

1. Los resultados muestran con valor negativo a los niños y jubilados.

2. Tiene problemas en la fundamentación teórica de la concepción económica de la sociedad.

Finalmente, Murphy y Topel (2006) plantean un modelo en donde se superan las limitantes del método del capital humano. No obstante, éste posee características que únicamente permiten su aplicación a países desarrollados.

\section{Desarrollo del modelo}

Primeramente se debe centrar la atención en analizar a un agente que con cierta probabilidad vive indefinidamente, y que en cada periodo de su vida debe decidir cuánto trabajar, consumir y acumular para periodos posteriores.

Cada individuo se coloca en un periodo determinado con una probabilidad exógena de sobrevivencia hacia el futuro. $\boldsymbol{S}_{\mathbf{0}}^{\mathbf{1}}$ es la probabilidad de sobrevivencia desde el nacimiento hasta el año siguiente, $\boldsymbol{S}_{\mathbf{1}}^{\mathbf{2}}$ es la probabilidad de sobrevivencia desde el año uno hasta el dos, y así sucesivamente. Por tanto, la probabilidad de sobrevivir hasta " $t$ " para un individuo con " $e$ " años de edad es:

$$
S_{e}^{t}=\prod_{i=e}^{t-1} S_{i}^{i+1}, \forall t>e
$$

A lo largo de su vida el agente obtiene utilidad por el consumo de bienes, ci, y el tiempo dedicado al ocio, $\boldsymbol{l}_{i}$ a través de la función de utilidad $U$ (ci,li), que es una función estrictamente creciente y cóncava en consumo y ocio. Así mismo, el individuo descuenta su utilidad de acuerdo a un factor de descuento $0<\boldsymbol{\beta}<1$. De esta manera, un agente cuya edad es " $e$ ", maximiza la siguiente función de utilidad.

$$
\sum_{i=e}^{\infty} U\left(c_{i}, l_{i}\right) \beta^{i-e} S_{e}^{i}
$$

Al comienzo de cada periodo cada individuo posee una dotación " $L$ " de unidades de tiempo que se pueden consumir en ocio o se pueden ofrecer en el mercado de trabajo, en cuyo caso se obtiene un retorno wi por unidad de tiempo. Por otro lado, el individuo obtiene un retorno del mercado de capitales a una tasa "r" por el nivel de activos $\boldsymbol{a} i$, depositados en el mercado al final del periodo pasado. Ambas fuentes de ingresos se pueden disponer en el periodo posterior para el consumo o se pueden acumular como activos en el futuro. 


$$
c_{i}+a_{i+1} \leq\left(1+r_{i}\right) a_{i}+\left(L-l_{i}\right) w_{i}
$$

En este ejercicio la única manera de trasferir recursos a futuro es a través de mercados de capitales. Es así que este modelo supone la imposibilidad de existencia de aseguramiento perfecto, contrario a trabajos previos para países desarrollados (Murphy \& Topel, 1999). De igual manera, de acuerdo a la estructura del mercado de capitales, si una persona muere, sus activos pasan a ser propiedad del intermediario financiero. Adicionalmente, se supone que el intermediario financiero no paga premio por riesgo de mortalidad, como por ejemplo ocurriría si existieran seguros de vida, todo esto teniendo en cuenta que existe una baja cobertura de estos instrumentos en Nicaragua. Es así que este modelo toma en cuenta dicha realidad y se centra en un tipo de mercado de capital adecuado para el caso de países en vías de desarrollo.

Para maximizar la utilidad sujeta a la restricción presupuestaria se plantea el Lagrangiano:

$L_{e}=\max _{\left\{c_{i}, l_{i}, a_{i+1}\right\}} \sum_{i=e}^{\infty}\left\{U\left(c_{i}, l_{i}\right) \beta^{i-e} S_{e}^{i}+\lambda_{i}\left[\left(1+r_{i}\right) a_{i}+(L-1) w_{i}-c_{i}-a_{i+1}\right]\right\}$

Donde $\lambda \boldsymbol{i}$ es el precio sombra de los recursos del periodo $\boldsymbol{i}$. En este caso se busca elegir una senda óptima de consumo, ocio y activos. Para ello las condiciones de primer orden son:

$$
\begin{array}{r}
U_{c}(i) \beta^{i-e} S_{e}^{i}=\lambda_{i}(4) \\
U_{l}(i) \beta^{i-e} S_{e}^{i} \geq \lambda_{i} w_{i} \\
-\lambda_{i}+\lambda_{i+1}\left(1+r_{i+1}\right)=0
\end{array}
$$

La ecuación (4) iguala a la utilidad marginal esperada del consumo, en $\boldsymbol{i}$ con su costo marginal $\lambda i$ La ecuación (5) tiene una interpretación análoga para el ocio, mientras que la ecuación (6) es la condición de optimalidad para la acumulación de activos.

\subsection{El valor económico de la vida}

Para conocer los beneficios de realizar una política económica de capital humano enfocada en el sector salud, se debe determinar cuál es el valor económico de la vida de un agente. En este punto se busca calcular cuál es la disponibilidad a pagar (DAP) que permita aumentar marginalmente la probabilidad de sobrevivencia desde el periodo $\boldsymbol{v}$ hasta el $\boldsymbol{t}$, donde $\boldsymbol{t}>\boldsymbol{v}$, esto es:

$$
\frac{\partial L}{\partial S_{v}^{t}}=\sum_{\tau=t}^{\infty} U\left(c_{\tau}, l_{t}\right) \beta^{t-e} \frac{\partial S_{e}^{\tau}}{\partial S_{v}^{t}}(7)
$$


Por tanto, es apreciable que el aumento en la utilidad del agente al aumentar marginalmente la tasa de supervivencia entre $\boldsymbol{v}$ y $\boldsymbol{t}$, tiene que ver con los flujos futuros de utilidad descontada con el factor de descuento $\boldsymbol{\beta}$ y ponderado de acuerdo a la posibilidad de supervivencia en cada periodo. ${ }^{1}$

La ecuación (7) está medida en niveles de utilidad y de ella se puede obtener inmediatamente el valor monetario al dividirse por el precio sombra en $\boldsymbol{t}$, de tal manera que:

$$
\frac{\partial L_{e}}{\partial S_{v}^{t}} /_{\lambda_{t}}=\sum_{\tau=t}^{\infty} \frac{U\left(c_{\tau}, l_{t}\right)}{\lambda_{t}} \beta^{t-e} \frac{\partial S_{e}^{\tau}}{\partial S_{v}^{t}}(8)
$$

Dado que esta expresión cumple con la propiedad de homogeneidad de grado $d$ en la función de utilidad, donde $0<d \leq 1$. Es decir:

$$
U\left(c_{\tau}, l_{\tau}\right)=\frac{1}{d}\left[U_{c}(\tau) c_{\tau}+U_{l}(\tau) l_{\tau}\right]=\frac{U\left(c_{\tau}, l_{\tau}\right)}{U_{c}\left(c_{t}, l_{t}\right)}=\frac{1}{d}\left[\frac{U_{c}(\tau)}{U_{c}(t)} c_{\tau}+\frac{U_{l}(\tau)}{U_{c}(t)} l_{\tau}\right]
$$

de las condiciones de primer orden se obtiene:

$$
\begin{gathered}
\frac{U_{c}(\tau)}{U_{c}(t)}=\frac{\lambda_{\tau}}{\lambda_{t}} \frac{\beta^{t-\tau}}{S_{t}^{\tau}}(9) \\
\frac{U_{l}(\tau)}{U_{l}(t)}=\frac{\lambda_{\tau} w_{\tau}}{\lambda_{t}} \frac{\beta^{t-\tau}}{S_{t}^{\tau}}(10) \\
\frac{\lambda_{t}}{\lambda_{\tau}}=\prod_{X=t+1}^{\tau} \frac{1}{\left(1+r_{x}\right)}(11)
\end{gathered}
$$

En este caso las dos primeras ecuaciones (9) y (10) son iguales entre tasas marginales de sustitución y precios relativos entre consumo en los periodos $\boldsymbol{t}$ y $\boldsymbol{t}$ y entre ocio en el periodo $\boldsymbol{t}$ y el consumo en el periodo $\boldsymbol{t}$ respectivamente. Asimismo, la ecuación (11) indica que el precio sombra de los activos cae a la tasa de rendimiento del mercado. ${ }^{2}$

Sustituyendo (8) en (11) tomando en cuenta la condición de homogeneidad en grado $d$ en la función de utilidad, es posible establecer el valor monetario de la vida como $^{3}$ :

Es importante notar que en la ecuación 7 aparecen todos los flujos futuros porque el cambio marginal de la
probabilidad de sobrevivencia entre v y t aumenta todas las probabilidades de sobrevivencia posteriores a v.
Esto se debe a una simple condición de arbitraje que establece que la ganancia de capital de los activos
(medida por su crecimiento en precios sombras) más la ganancia en rentabilidad de mantenimiento de los
activo debe ser cero. Si esta condición no se cumpliera, los individuos preferirían mantener más activos, en
el caso en que la ganancia de capital más la rentabilidad fuese positiva. Si fuese negativa, los individuos
preferirian mantener menos activos.
Se está suponiendo que el efecto de la supervivencia sólo afecta el periodo de v a t y lo hace proporcionalmente.
Es así que:

$$
\frac{\partial S_{e}^{\tau}}{\partial S_{v}^{t}} \frac{1}{S_{e}^{\tau}}=\frac{S_{e}^{v} S_{t}^{\tau}}{S_{e}^{\tau}}
$$

En este caso, esta expresión se calcula como incremento finito, de esta manera:

$$
\frac{S_{e}^{v} S_{v}^{-j, \tau}-S_{e}^{v} S_{v}^{\tau}}{S_{e}^{\tau}}
$$

Donde $S_{v}^{-j, \tau}$ es la supervivencia modificada por la erradicación de algunas enfermedades tipo $j$. 
Esta última ecuación (12) indica que el valor de aumentar marginalmente la probabilidad de sobrevivencia entre $\boldsymbol{v}$ y $\boldsymbol{t}$ depende de una serie de factores, dígase:

- $\quad$ En primer lugar, el valor presente de todo el patrón de gasto a realizarse desde el periodo $t$ en adelante, descontado a la tasa de interés del mercado. Este patrón de gasto incluye tanto los gastos en bienes de consumo como el gasto realizado en ocio, esto es el gasto total del individuo. Es así que se incluye el "gasto total" desde $t$ en adelante, porque un aumento en la probabilidad de supervivencia entre $\boldsymbol{v}$ y $\boldsymbol{t}$ influye directamente en las probabilidades de supervivencia posteriores y, por ello, también hace más probable los flujos de ingresos posteriores.

- En segundo lugar, este valor depende de la constante $d$, que refleja el grado de concavidad de la función de utilidad. De esta manera, mientras menor es $d$, mayor es el valor económico de la vida. La razón es que mientras más cóncava sea la función de utilidad, los individuos desean menores fluctuaciones en sus patrones de gasto y, por tanto, valoran más los periodos adicionales de vida cuyos valores de consumo son más parecidos a los anteriores.

Finalmente, el valor depende del último factor de la ecuación $\frac{\partial \boldsymbol{S}_{e}^{\tau}}{\boldsymbol{\partial} \boldsymbol{S}_{v}^{t}} \frac{\mathbf{1}}{\boldsymbol{S}_{\boldsymbol{e}}^{\tau}}$. Este es el cambio en tasas de supervivencia debido a la implementación del programa de salud entre $\boldsymbol{v}$ y $\boldsymbol{t}$. En el caso particular donde el efecto de la supervivencia sólo afecta el periodo de $\boldsymbol{v}$ a $\boldsymbol{t}$ y lo hace proporcionalmente, se calcula como incremento finito, es decir: $\frac{\partial S_{e}^{\tau}}{\partial S_{v}^{t}} \frac{1}{S_{e}^{\tau}}=\frac{S_{e}^{v} S_{t}^{\tau}}{S_{e}^{\tau}}$. El impacto de los cambios en la tasa de supervivencia entre $v$ y $\boldsymbol{t}$ depende de aquellas tasas que no han cambiado, es decir, de las que están entre $\boldsymbol{e}$ y $\boldsymbol{v}$ y de $\boldsymbol{t}$ a $\boldsymbol{t}$. De esta manera, cuanto mayor sea la importancia relativa de estas probabilidades de supervivencia que no varían (es decir, las relacionadas con periodos en los que no se ha aplicado el programa de salud), mayor es el valor económico del programa. Existe complementariedad entre las tasas de supervivencia. Esto es claro si se toma en cuenta que un programa de salud de poco sirve si solamente aumenta la probabilidad de supervivencia por unos pocos periodos, pero con posterioridad existe certidumbre de fallecimiento. En contraposición, cuando la probabilidad de supervivencia posterior al programa es más alta, implementar el programa permitirá disfrutar con bastante más certidumbre de los flujos futuros.

La ecuación (12) será utilizada a continuación para realizar distintos ejercicios concernientes a determinar el valor económico de la vida en el caso nicaragüense. Con este propósito, la próxima sección se dedica a describir la forma de calibrar el modelo, mientras que la siguiente muestra los resultados.

\section{Aplicación del modelo}

\subsection{Calibración del modelo}

Para poder calibrar el modelo con el objetivo de encontrar el valor económico de la vida, se siguieron una serie de pasos. El primer paso fue determinar el patrón de gasto de las personas a través de su vida $\left(\boldsymbol{c}_{\boldsymbol{\tau}}+\boldsymbol{w}_{\boldsymbol{\tau}} \boldsymbol{l}_{\boldsymbol{\tau}}, \forall \boldsymbol{\tau}\right)$, que en este modelo tiende a $\frac{\boldsymbol{w}_{\boldsymbol{\tau}} \boldsymbol{l}_{\boldsymbol{t}}}{\boldsymbol{\alpha}}, \forall \boldsymbol{\tau}$. Una vez realizado este paso, el siguiente consistió en determinar la constante $\boldsymbol{d}$ y $\boldsymbol{a}$ que determinan la concavidad y el peso del ocio en la función de utilidad. Una vez cumplidos estos pasos, solamente restó el paso culminante, que 
consistió en encontrar una tasa de interés a la que se descuenten los flujos de gasto y la evolución de las probabilidades de supervivencia que determinan $\frac{\partial S_{e}^{\tau}}{\partial S_{v}^{t}} \frac{1}{S_{e}^{\tau}}$.

En este paso se utilizaron las encuestas de empleo 2000, 2003, 2004, 2005, 2006, 2007 y 2008 (INIDE, 2009). El objetivo del uso de dichas encuestas fue evaluar ciertas condiciones del mercado laboral en los últimos años, teniendo en cuenta diferentes tramos de edades que permitirían diferenciar grupos etarios que son más o menos vulnerables a diferentes tipos de enfermedades. Luego se procedió a determinar específicamente el salario promedio por edad de la población nicaragüense dividida por sexo (perfil de ingreso por edad en un momento determinado en el tiempo) con el objetivo de estimar el gasto en ocio. Esto se hizo concretamente con la encuesta de 2008.

En el Cuadro 1 se muestra la evolución de las horas trabajadas para diferentes grupos de edades y divididas por sexo durante el periodo 2000-2008. En ella se aprecia una estabilidad en cuanto a las horas trabajadas desde el año 2000. Además, tanto los hombres como las mujeres en promedio trabajan las mismas horas en el primer estrato de edad, es decir de los 15 a los 19 años. Luego de eso se observa que los hombres trabajan de los 20 a los 24 años en promedio alrededor de 50 horas y luego de los 25 a los 49 años 51 horas, mientras que las mujeres desde los 20 hasta los 49 años trabajan 47 horas. Esto quiere decir que, en promedio, en la mayor parte de la vida laboral de los nicaragüenses los hombres trabajan cuatro horas semanales más que las mujeres, y esta diferencia alcanza las cinco horas en promedio en el periodo que empieza a partir de los 50 años y en el cual culmina la vida laboral.

Cuadro 1. Horas promedio trabajadas en Nicaragua por sexo y grupo de edad, 20002008

\begin{tabular}{|c|c|c|c|c|c|c|c|c|c|c|c|c|}
\hline \multirow[b]{2}{*}{ Edad } & \multicolumn{12}{|c|}{ Horas Hombres } \\
\hline & $15-19$ & $20-24$ & $25-29$ & $30-34$ & $35-39$ & $40-44$ & $45-49$ & $50-54$ & $55-59$ & $60-64$ & 65 & y más \\
\hline & 2000 & 46 & 50 & 51 & 51 & 51 & 51 & 51 & 50 & 49 & 50 & 47 \\
\hline & 2003 & 42 & 48 & 50 & 49 & 51 & 50 & 50 & 50 & 48 & 46 & 44 \\
\hline & 2004 & 48 & 51 & 51 & 51 & 51 & 51 & 50 & 50 & 49 & 48 & 45 \\
\hline & 2005 & 46 & 50 & 51 & 52 & 52 & 51 & 51 & 50 & 49 & 46 & 46 \\
\hline & 2006 & 47 & 50 & 51 & 51 & 52 & 53 & 51 & 51 & 52 & 50 & 46 \\
\hline & 2007 & 47 & 49 & 50 & 52 & 51 & 50 & 49 & 51 & 50 & 46 & 46 \\
\hline & 2008 & 47 & 50 & 51 & 51 & 51 & 51 & 51 & 50 & 51 & 48 & 45 \\
\hline \multicolumn{13}{|c|}{ Mujeres } \\
\hline Edad & $15-19$ & $20-24$ & $25-29$ & $30-34$ & $35-39$ & $40-44$ & $45-49$ & $50-54$ & $55-59$ & $60-64$ & 65 & y más \\
\hline & 2000 & 46 & 48 & 48 & 48 & 48 & 48 & 49 & 48 & 49 & 42 & 42 \\
\hline & 2003 & 40 & 45 & 47 & 45 & 45 & 46 & 45 & 46 & 41 & 42 & 40 \\
\hline & 2004 & 46 & 48 & 47 & 47 & 48 & 47 & 46 & 45 & 44 & 40 & 42 \\
\hline & 2005 & 47 & 48 & 48 & 47 & 48 & 48 & 48 & 46 & 44 & 43 & 40 \\
\hline & 2006 & 48 & 48 & 48 & 46 & 47 & 47 & 47 & 46 & 47 & 42 & 40 \\
\hline & 2007 & 45 & 47 & 46 & 46 & 48 & 48 & 46 & 44 & 45 & 42 & 38 \\
\hline & 2008 & 49 & 48 & 48 & 46 & 47 & 47 & 45 & 45 & 43 & 41 & 40 \\
\hline
\end{tabular}

Fuente: Elaboración propia en base a Encuesta de Medición de Empleo (EME) 2000, 2003, 2004, 2005, 2006, 2007 y 2008 (INIDE, 2009). 
En el Cuadro 2 se observa la evolución del salario real en Nicaragua por estrato de edades y dividido por sexo en el periodo 2000-2008. El análisis de estos datos muestra que cuando tanto hombres como mujeres pasan del primer estrato de edad (15-19 años) al segundo (20-24 años) experimentan la mayor variación de sus ingresos a lo largo de su vida ya que el salario real aumenta en promedio para hombres y mujeres $56.38 \%$ y $52.81 \%$ respectivamente. A partir de ese momento se experimentan sucesivos pero desacelerados aumentos del salario real tanto para hombres como para mujeres, aunque para las mujeres estos aumentos del salario se detienen en el estrato que va de los 45 a los 49 años, donde el salario empieza a experimentar decrecimientos sucesivos empezando por una caída de $2.06 \%$ en dicho estrato seguido por caídas de $3.53 \%, 14.71 \%, 22.10 \%$ y $8.09 \%$ en los estratos siguientes. Por otro lado, para los hombres, a diferencia de las mujeres, la caída de los salarios reales se empieza a manifestar hasta el estrato que va de los 55 a los 59 años.

En lo que respecta a la diferencia de remuneración entre hombres y mujeres, en promedio a lo largo de la vida los hombres ganan un $45.13 \%$ más que las mujeres y estas diferencias salariales se manifiestan desde el primer estrato de edad (15-19 años), donde la diferencia es solamente de $16.52 \%$. Esta brecha se ensancha a lo largo de los siguientes estratos de tal forma que de manera sucesiva se observa cada vez una mayor diferencia hasta llegar al estrato que va de los 60 a los 64 años, donde la diferencia llega a ser de un $80.65 \%$. Finalmente, en el último estrato (mayores de 65 años) se observa que cae hasta $66.44 \%$. 

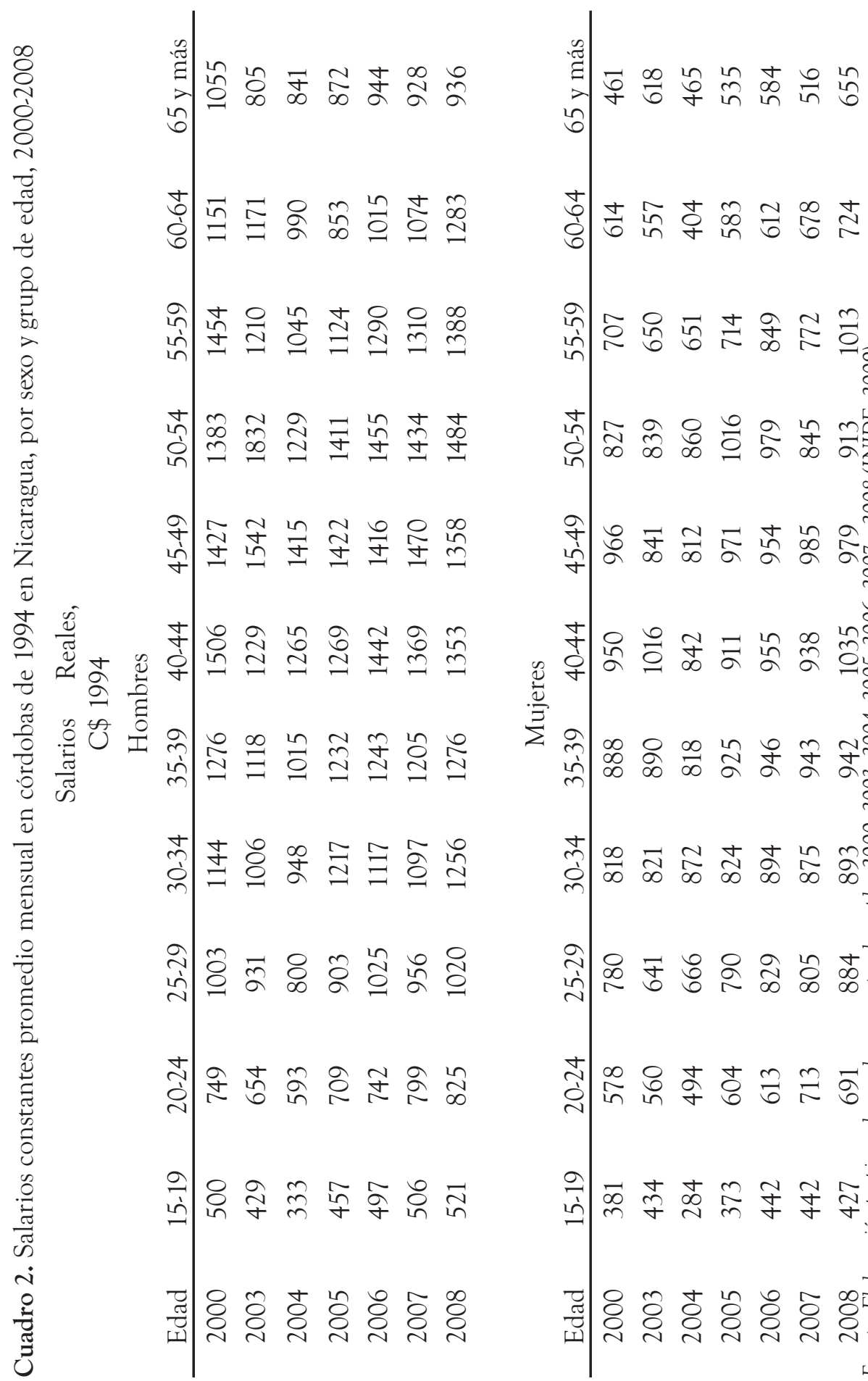

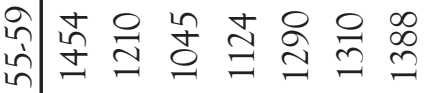

ஸे กิ

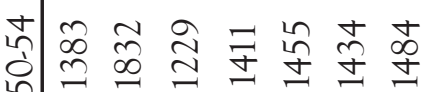

ท่ำ

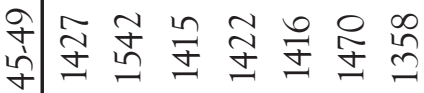

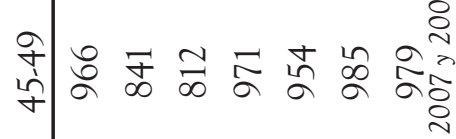

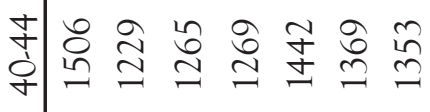

กิ

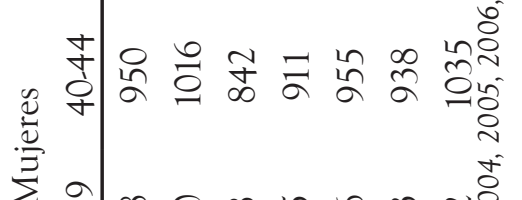

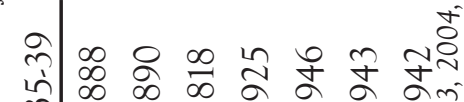

๖ீ๋ ๖

m

ิํํำ

ヘิ๋

ホั๋

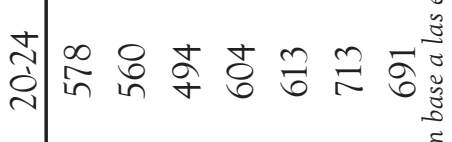

ำ

ทे

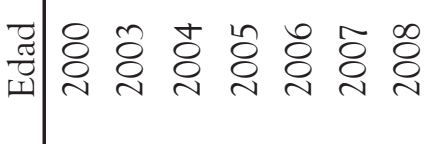

I 
Como antes se mencionó, en este caso las estimaciones referentes al gasto en ocio se basan en la Encuesta de Medición de Empleo de 2008 (INIDE, 2009). Se pretende calcular el costo de oportunidad del ocio a través de estimaciones de ingreso laboral por medio de una estimación de tipo Mincer (1974). De esta manera también se podrá obtener una aproximación de salario por hora que permitirá estimar el valor del ocio.

La encuesta considerada fue realizada a individuos durante el año 2008. Es una encuesta que únicamente incluye preguntas del ámbito laboral. Esta base de datos proporciona la información necesaria para estimar las ecuaciones de tipo Mincer (1974), que utiliza como variable dependiente el ingreso (nominal) laboral de los trabajadores y como variables independientes, la edad, la edad al cuadrado, los años de educación y el sexo de las personas. Adicionalmente, es importante mencionar que esta ecuación fue estimada para personas mayores a 15 años, así también, es necesario recalcar que se incluyen dummies para los individuos entre 65 y 75 años, 70 y 75 años, 75 y 80 años y mayores a 80 años que interactúan con la variable "edad". Así mismo, en esta ecuación se corrigió el sesgo de selección de la entrada a la fuerza de trabajo por el conocido método de dos etapas de Heckman (1979), y de esa manera se concluyó que en el año 2008 no existe evidencia de este problema en la estimación.

En la Ilustración 1 se muestra la estimación de la evolución de los ingresos laborales de hombres y mujeres de 11 y 16 años de escolaridad. En dicho gráfico se aprecia una clara diferencia en los ingresos laborales entre hombres y mujeres para un mismo nivel de educación. Así mismo se aprecia una clara diferencia de ingresos entre individuos del mismo sexo pero con diferentes niveles de educación.

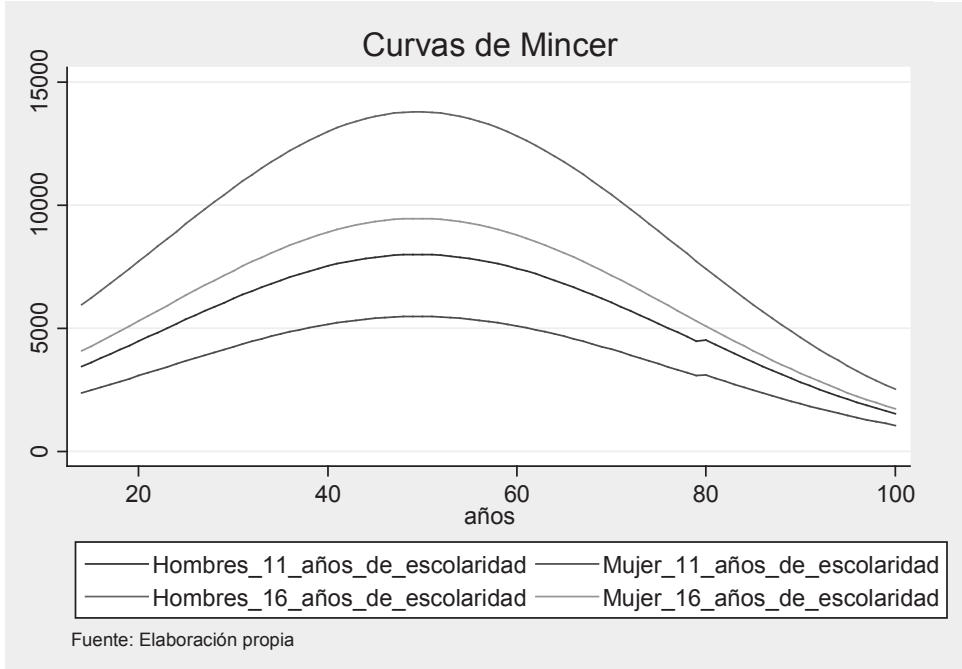

Ilustración 1. Evolución estimada de ingresos laborales a través del ciclo de vida, C\$ 2008

Una vez determinada la evolución de los ingresos mensuales laborales a través del ciclo de vida, se procede a estimar el valor del ocio de la siguiente manera: se asume que el número de horas trabajadas por semana es de 48, esta suposición es 
muy ajustada a la realidad nacional. Esto se puede apreciar a través de la Ilustración 2, que muestra la distribución de las horas trabajadas por semana por parte de los trabajadores nicaragüenses en 2008. En dicho gráfico se observa que la distribución tiene una media de 48 horas y una distribución estándar de solamente 15.76 horas. Esto es consecuencia de que poco más del $27 \%$ de los individuos reportaron trabajar 48 horas por semana.

Una vez establecido este supuesto, se procede a calcular el salario por hora a través del ciclo de vida, utilizando las estimaciones anteriores y dividiéndolas por 192 horas $^{4}$. Con esta estimación del salario por hora se determina el gasto en ocio multiplicando salario por hora a través del ciclo de vida por 480 horas 5 .

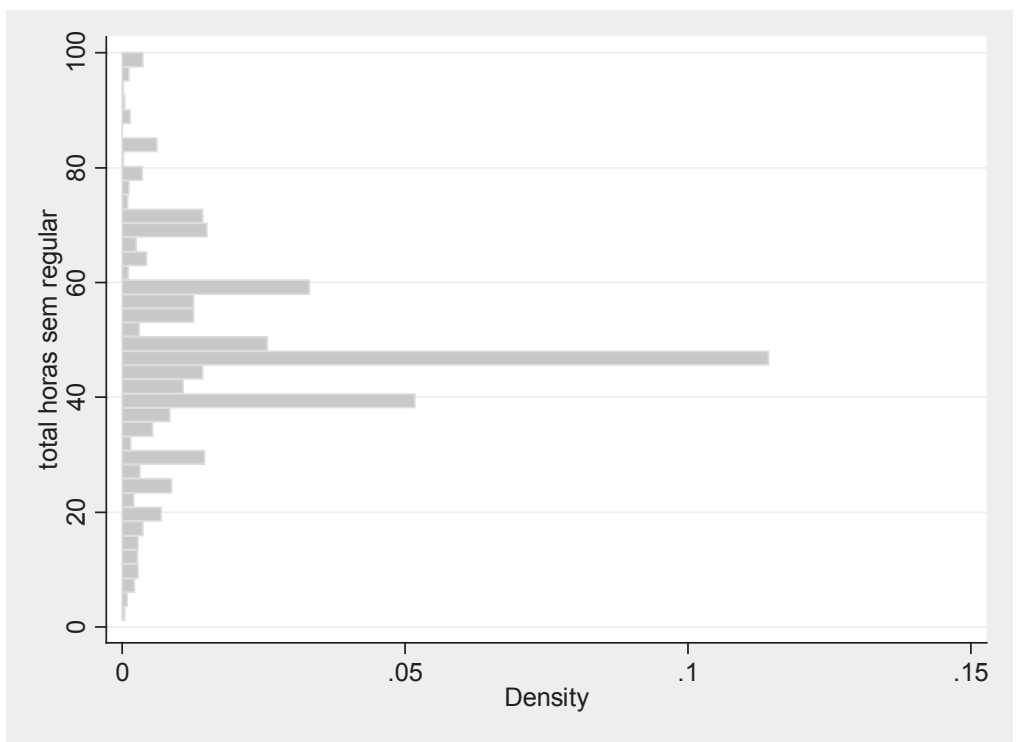

Ilustración 2. Distribución de horas trabajadas por semana, 2008

El paso siguiente es calibrar las probabilidades de supervivencia y el parámetro $\boldsymbol{d} \boldsymbol{\alpha}$. Con el propósito de calibrar el parámetro $\boldsymbol{d} \boldsymbol{\alpha}$ se sigue la siguiente estrategia: primeramente es necesario establecer que el valor económico de la vida estadística al nacer es el aumento en el nivel de ingresos inicial, $d a_{0}$, que compensa por el aumento en el riesgo de mortalidad en todos los periodos posteriores, $d S^{t}{ }_{e}=d S, \forall t$, tal que el nivel de utilidad esperada del individuo no cambie, $d L_{0}=0$. Es decir:

$$
d L_{0}=\sum_{i=0}^{\infty}\left(u\left(c_{i}, l_{i}\right) \beta^{i} d S_{0}^{i}\right)+\lambda_{0} d a_{0}
$$

Dado que, $\boldsymbol{d} \boldsymbol{S}_{\mathbf{0}}^{\boldsymbol{i}}=\boldsymbol{d} \boldsymbol{S}, \forall \boldsymbol{i}$, entonces:

$$
\frac{d a_{0}}{d S}=-\frac{\sum_{i=0}^{\infty} u\left(c_{i}, l_{i}\right) \beta^{i}}{\lambda_{0}}=-\frac{\sum_{i=0}^{\infty} u\left(c_{i}, l_{i}\right) \beta^{i}}{u_{c}\left(c_{0}, l_{0}\right)}
$$

48 horas por semana por cuatro semanas al mes.

7 dias a la semana por cuatro semanas al mes, menos 192 horas trabajadas. 
Introduciendo (9) en (11), (13) y suponiendo una tasa de interés constante a través del ciclo de vida. $r_{x}=r, \forall x$, se obtiene en definitiva.

$$
\frac{d a_{0}}{d S}=-\frac{1}{d \alpha} \sum_{i=0}^{\infty}\left[\frac{1}{S_{0}^{i}} \frac{w_{i} l_{i}}{(1+r)^{i}}\right]
$$

En el caso de Nicaragua existe cierta evidencia del valor de $\frac{d a_{0}}{d s}$. La Conferencia Interamericana de Seguridad Social (CISS, 2009) reporta que el valor de la vida estadística en Nicaragua en 2009 era de alrededor de 126,884 dólares de 2006 $(2,229,351.88$ córdobas de 2006). Es así que en este trabajo se asume ese valor para

$\frac{\partial \boldsymbol{a}_{\mathbf{0}}}{\boldsymbol{\partial} \boldsymbol{S}}$. Con esta información, así como con las anteriores estimaciones del valor del ocio y las tablas de mortalidad, se procede a calcular los parámetros .

\subsection{Probabilidades de supervivencia}

Las probabilidades de supervivencia se obtuvieron a partir de las tablas anuales de vida, las cuales están conformadas por la población total $\left(\boldsymbol{P}_{\chi}\right)$, número de defunciones $\left(D_{\chi}\right)$, probabilidades de morir $\left(q_{\chi}\right)$, número de sobrevivientes en cohorte hipotético de 100,000 nacidos vivos $\left(\mathbf{L}_{x}\right)$ y probabilidades de sobrevivir $\left(\boldsymbol{S}_{\chi}\right)$. Todo esto es determinado para individuos de 0 a 100 años dividido por sexo.

Para la obtención de las probabilidades de supervivencia por edad de los individuos se obtuvo información de la población y defunciones por grupos de edad y divididas por sexo. Dicha información fue facilitada por la oficina de estadísticas del MINSA-Central y el INIDE. En esta información se describe la mortalidad por grupos de edades en intervalos de cinco años desde la fecha de nacimiento hasta los 100 años de edad.

En el modelo utilizado se requiere calcular el valor económico de la vida año a año, por lo que resulta necesario construir tablas anuales. Es para ello que se utilizó el método de construcción de tablas anuales de vida (anual life tables) del National Center for Health Statistics (1999). En este método se calculan interpolaciones anuales que se denominarán por $\boldsymbol{P}_{\chi}$ y $\boldsymbol{D}_{\chi}$, donde $\boldsymbol{P}_{\chi}$ es la población de edad $\boldsymbol{X}_{\mathrm{y}} \boldsymbol{D}_{\boldsymbol{X}}$ es el número de defunciones para población con edad $X$. Los valores $P_{X}$ se obtienen por interpolación utilizando la formula de Beer:

$$
\boldsymbol{P}_{\mathbf{0}+k}=C_{k, 05} \boldsymbol{P}_{0}+C_{k, 55} \boldsymbol{P}_{5}+C_{k, 105} \boldsymbol{P}_{10}+C_{k, 155} \boldsymbol{P}_{15}+C_{k, 205} \boldsymbol{P}_{20}
$$

En este caso, es la población de edad $\mathrm{X}+\mathrm{k}$ (donde $\mathrm{k}=0,1,2,3,4)$, 5Px es la población total con edad entre $\mathrm{X}, \mathrm{y} \mathrm{X}+5$ y son los coeficientes de interpolación de Beer que se muestran en el Cuadro 3. Para interpolar los valores de 0 a 5 años, de 5 a 95 años y de 95 a 100 años, se aplican las formulas:

$$
\begin{gathered}
P_{0+k}=C_{k, 05} P_{0}+C_{k, 55} P_{5}+C_{k, 105} P_{10}+C_{k, 155} P_{15}+C_{k, 205} P_{20} \\
P_{5+k}=C_{k, 05} P_{0}+C_{k, 55} P_{5}+C_{k, 105} P_{10}+C_{k, 155} P_{15}+C_{k, 205} P_{20} \\
P_{95+k}=C_{k, 805} P_{80}+C_{k, 855} P_{85}+C_{k, 905} P_{90}+C_{k, 955} P_{95}+C_{k, 100} P_{100}
\end{gathered}
$$

La interpolación para todos los grupos de edades es similar con la excepción de que al utilizar el coeficiente de Beer para interpolar las edades de 5 a 9 y de 10 a 
14 años se utiliza un valor ficticio ${ }^{6}$ de 5D0. Este es un valor ficticio del número de defunciones en el intervalo de 0 a 4 años de edad. Esto es así ya que al realizar el ejercicio de la interpolación, los números obtenidos para las edades de 5 a 14 años son discontinuos en relación a los números obtenidos para las edades entre 0 y 4 años de edad.

Cuadro 3. Coeficiente de Beer para realizar interpolaciones

\begin{tabular}{|cccccc|}
\hline 0 a 4 años & 0 & 5 & 10 & 15 & 20 \\
\hline 0 & 0.3333 & -1.636 & -0.01 & 0.0796 & -0.0283 \\
1 & 0.2595 & -0.078 & 0.013 & 0.01 & -0.0045 \\
2 & 0.1924 & 0.0064 & 0.0184 & -0.0256 & 0.0084 \\
3 & 0.1329 & 0.0844 & 0.0054 & -0.0356 & 0.0129 \\
4 & 0.0819 & 0.1508 & -0.0158 & -0.0284 & 0.0115 \\
\hline 5 a 9 años & 0 & 5 & 10 & 15 & 20 \\
\hline 5 & 0.0404 & 0.2 & -0.0344 & -0.0128 & 0.0068 \\
6 & 0.0093 & 0.2268 & -0.0402 & 0.0028 & 0.0013 \\
7 & -0.0108 & 0.2272 & -0.0248 & 0.0112 & -0.0028 \\
8 & -0.0198 & 0.1992 & 0.0178 & 0.0072 & -0.0038 \\
9 & -0.0191 & 0.1468 & 0.0822 & -0.0084 & -0.0015 \\
\hline $\mathrm{x}$ a x+5 años & $\mathrm{x}-10$ & $\mathrm{x}-5$ & $\mathrm{X}$ & $\mathrm{x}+5$ & $\mathrm{x}+10$ \\
\hline $\mathrm{X}$ & -0.0117 & 0.0804 & 0.157 & -0.0284 & 0.0027 \\
$\mathrm{x}+1$ & -0.002 & 0.016 & 0.22 & -0.04 & 0.006 \\
$\mathrm{x}+2$ & 0.005 & -0.028 & 0.246 & -0.028 & 0.005 \\
$\mathrm{x}+3$ & 0.006 & -0.04 & 0.22 & 0.016 & -0.002 \\
$\mathrm{x}+4$ & 0.0027 & -0.0284 & 0.157 & 0.0804 & -0.0177 \\
\hline $95 \mathrm{a} 99$ años & 80 & 85 & 90 & 95 & 100 \\
\hline 95 & -0.0015 & -0.0084 & 0.0822 & 0.1468 & -0.0191 \\
96 & -0.0038 & 0.0072 & 0.0172 & 0.1992 & -0.0198 \\
97 & -0.0028 & 0.0112 & -0.0248 & 0.2272 & -0.0108 \\
98 & 0.0013 & 0.0028 & -0.0402 & 0.2268 & 0.0093 \\
99 & 0.0068 & -0.0128 & -0.0344 & 0.2 & 0.0404 \\
\hline
\end{tabular}

Una vez realizado este paso el siguiente es estimar primeramente la probabilidad de una persona de morir a los $\mathrm{X}$ años, $q_{X}$, en el tramo de edad de $\mathrm{X}$ a $\mathrm{X}+1$. Luego se debe determinar el número de sobreviviente de edad $\mathrm{X}$ de un cohorte de 100,000 nacidos vivos, que se denominará $L_{x}$.

Se estima $L_{x}$ de la forma: $L_{x}=L_{x, 1}\left(1-q_{x, 1}\right)$, donde $L_{0} 100,000$. Pero antes se debe determinar $\boldsymbol{q}_{X}$ Ésta se determinará de la forma: 


$$
\boldsymbol{q}_{x}=\frac{\boldsymbol{D}_{x}}{\boldsymbol{P}_{x}+\frac{1}{2} D_{x}}
$$

Sin embargo, se debe tener cuidado con las probabilidades de fallecer de los menores a un año. Esto se debe a que las defunciones de este grupo de edad corresponden a nacidos en el año 2008 y defunciones del año anterior. Es por ello que se utiliza un factor de separación, $f$, que indica la proporción de los nacidos el año anterior pero fallecidos este año. Es así que la probabilidad de fallecimiento para este grupo de edad se calcula:

$$
q_{x}=\frac{D_{0}(1+f)}{B^{t}}+\frac{D_{0} f}{B^{t-1}}
$$

Donde $B^{t}$ y $B^{t-1}$ son el numero de nacidos en el año 2008 y el anterior, respectivamente. En este caso, de los datos de mortalidad de menores de un año reportados se calcula un $f=0.55$. Es así que se tiene la información necesaria para determinar la probabilidad de fallecimiento, $L_{X}$, y luego a partir de ésta la probabilidad de supervivencia, que se denomina por $S_{x}$.

\section{Determinación del beneficio económico social}

El primer ejercicio a resolver es la cuantificación del valor económico de la vida. Primeramente se determinará la disponibilidad a pagar por aumentos de la probabilidad de supervivencia sin tomar en cuenta una política de salud en particular que afecte directamente a una enfermedad específica. Por lo tanto, en este primer ejercicio no se especificará la causa (enfermedad combatida) de la disminución en la tasa de mortalidad. Para ello se debe estimar la ecuación (14), asumiendo que $\boldsymbol{v}=t-1$, con motivo de tomar en cuenta las diferencias tanto en capital humano como la discriminación imperante en Nicaragua. Se debe realizar este ejercicio para distintos individuos, con diferentes niveles educativos (primaria completa, secundaria completa y universidad completa) y diferente sexo.

Los resultados indican que el valor económico de la vida alcanza su máximo aproximadamente a los 43 años (véase Ilustración 3). Pero es notorio que existe una diferencia sustancial entre los individuos del mismo sexo pero con diferentes grados de escolaridad, además de una diferencia aún más marcada entre individuos de diferente sexo a tal punto que un hombre con 11 años de escolaridad (secundaria completa) reporta casi el mismo valor que una mujer con 16 años de escolaridad (universidad completa), y los hombres con 6 años de escolaridad (primaria completa) reportan un valor mayor a las mujeres con 11 años de escolaridad (secundaria completa). Esto se debe a que las personas con menor escolaridad y las mujeres tienen un menor patrón de ingresos laborales a lo largo del ciclo de vida, y por consiguiente el costo de oportunidad de su ocio es menor. 


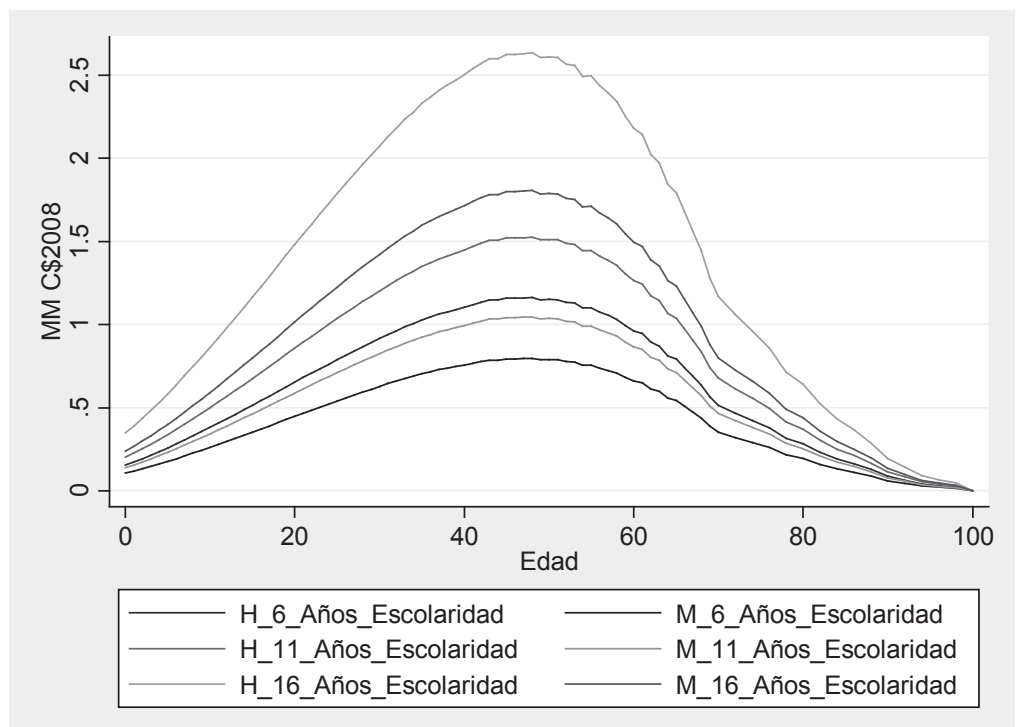

Ilustración 3. Valor de vida, Nicaragua MMC $\$ 2008$

\subsection{Valor económico de erradicar enfermedades especificas}

En este punto se obtendrá el valor monetario que producirían programas de salud que incidan en las tasas de mortalidad de las enfermedades que más afectan a la población nicaragüense. Dichos programas pueden ser de carácter preventivo o curativo, de tal modo que las familias puedan obtener beneficios adicionales como resultado de la disminución en los costes de tratamiento. En 2008 fallecieron 10,476 hombres y 7,765 mujeres. Esto representa una tasa bruta de mortalidad de 4.8 por cada mil habitantes; de 5.4 para hombres y 4.2 para mujeres. El $30.48 \%$ de la totalidad de los fallecimientos se deben a cinco enfermedades y a accidentes de tránsito. Esta última causa de muerte se incluye por la gran importancia que tiene a nivel nacional, siendo la sexta causa de muerte más común. Por otro lado, las enfermedades a tratar son: infarto agudo de miocardio, insuficiencia renal crónica, accidente vascular encefálico agudo ${ }^{7}$, neumonía y enfermedades pulmonares obstructivas crónicas.

El Cuadro 3 muestra el desglose de estas causas de fallecimiento. En él se puede apreciar que las causas de muerte para hombres y mujeres difieren considerablemente en algunas enfermedades y en otras no. También se observa que la principal causa de muerte en ambos sexos es el infarto agudo de miocardio. 
Cuadro 3. Principales causas de fallecimiento, Nicaragua 2008, número de defunciones

\begin{tabular}{c|cc|} 
Causas de fallecimiento & Hombres & Mujeres \\
\hline Infarto agudo de miocardio & 907 & 748 \\
Insuficiencia renal crónica & 611 & 199 \\
Acc. vascular encefálico agudo & 336 & 330 \\
Neumonía & 521 & 418 \\
Enfermedad pulmonar obstructiva crónica & 296 & 245 \\
Accidentes de tránsito & 301 & 47 \\
\cline { 2 - 3 }
\end{tabular}

Fuente: Oficina de estadísticas del MINSA-Central.

La Ilustración 4 descompone las causas de muerte de acuerdo a grupos de edades. De este gráfico se puede desprender que todas las causas de muerte, con excepción de los accidentes de tránsito (excluido del gráfico porque afectan a individuos de modo aleatorio), son muy incidentes en los individuos mayores a 40 años. Sin embargo, sólo la neumonía es importante para individuos menores a 22 años.

A continuación se determinó el beneficio de programas de salud que erradiquen la mortalidad por las causas antes mencionadas ${ }^{8}$. Cuando se erradica cada una de las causas de fallecimiento y se mantienen constantes las demás, las probabilidades de supervivencia cambian. De este modo, el valor económico de la vida entre estos dos casos varía debido a estas probabilidades. Es por ello que para evaluar los beneficios del programa, se procedió a construir tablas anuales suponiendo que se erradica cada enfermedad, mientras las demás se mantienen contantes.

Dado que se determinó el valor de erradicar la mortalidad de cada una de las enfermedades, en términos de notación se denominó $S_{v}^{-j, t}$ como la probabilidad de supervivencia entre v y t, una vez que se erradicó la enfermedad j. Los Cuadros 4 a 10 (ver en el Anexo) muestran este ejercicio para las causas de muerte en estudio.

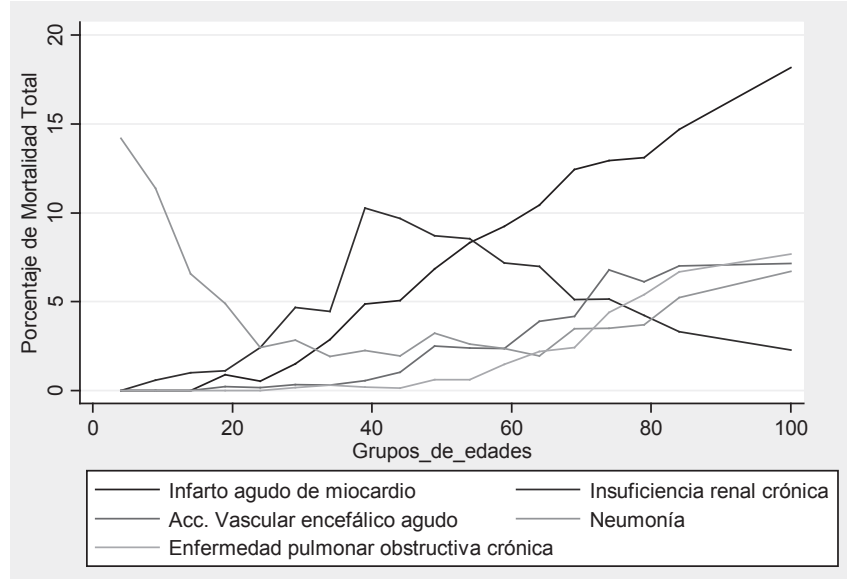

Ilustración 4. Causas específicas de muerte como porcentaje de la mortalidad total

De este modo también es posible determinar los beneficios sociales de programas que disminuyan la mortalidad de cualquier enfermedad en un monto determinado. 
Una vez efectuado este ejercicio, se construye el beneficio del programa o la disponibilidad a pagar, es así que se determinó:

$$
\Delta\left(\frac{\partial \boldsymbol{L}_{e}^{-j}}{\boldsymbol{\partial} \boldsymbol{S}_{v}^{t}} \frac{\mathbf{1}}{\lambda_{t}}\right)=\frac{1}{d \alpha} \sum_{\tau=t}^{\infty}\left\{\left(\prod_{x=t+1}^{\tau} \frac{1}{\left(1+r_{x}\right)}\right) w_{\tau} l_{\tau}\right\}\left(\frac{S_{e}^{v} S_{v}^{-j, \tau}}{S_{e}^{\tau}}-\frac{S_{e}^{v} S_{v}^{\tau}}{S_{e}^{\tau}}\right)
$$

Es decir, la disponibilidad a pagar por erradicar la enfermedad j es simplemente la diferencia entre la disponibilidad a pagar por aumentos de vida bajo dos regímenes diferentes de supervivencia (antes y después el programa de salud).

Las ganancias por erradicar cada una de estas causas de muerte para individuos con diferentes niveles educativos y sexo se muestran en las Ilustraciones 5 a 10. La Ilustración 5 muestra el caso de erradicar muertes por infarto agudo de miocardio. La disponibilidad a pagar aparece desde los 45 años y crece significativamente hasta que empieza a disminuir a los 91 años. La disponibilidad a pagar máxima por esta enfermedad es mucho mayor a las demás, esto se debe a que las tasas de mortalidad por esta enfermedad son mucho mayores a las demás.

Otro caso interesante es la neumonía, que muestra una gran disponibilidad a pagar al ser erradicada para individuos mayores a 80 años mientras que existe una pequeña disponibilidad a pagar por individuos menores a 20 años. Esto se debe a que la neumonía representa aproximadamente el diez por ciento de la mortalidad de individuos menores de 20 años. Es por ello que erradicarla en ese grupo de edad no reporta gran ganancia, ya que las tasas de mortalidad en este grupo son tan pequeñas que una caída adicional del diez por ciento tiene un efecto relativamente pequeño. Por otro lado, la neumonía representa aproximadamente el quince por ciento de la mortalidad en los individuos mayores a 80 años. Sin embargo, en este grupo hay una enorme disposición pagar debido a que existe una alta mortalidad año a año en su rango de edad. Se observa una situación similar para la insuficiencia renal crónica, accidente vascular encefálico agudo y enfermedad pulmonar obstructiva crónica.

En cuanto a los accidente de tránsito, se observa una baja disponibilidad máxima a pagar en relación a las demás causas de muerte. Esto se debe a que esta causa es la que menos afecta a la población en comparación con las demás.

\subsection{Beneficio económico de erradicar enfermedades especificas como porcentaje del PIB}

Hasta el momento se ha determinado cuál es el valor económico de la vida para agentes individuales que variaban de acuerdo a su edad, sexo y nivel educativo. En este punto se procede a describir el valor total para la sociedad de erradicar alguna de estas causas de muerte en Nicaragua. Es por ello que en esta sección se presenta cuánto es el valor agregado de erradicar dichas causas de muertes.

Los Cuadros 11 y 12 revelan los resultados. En ellos se observa el cálculo de los beneficios per cápita para hombres y mujeres y por edad, de erradicar las causas de fallecimiento. Así mismo se muestra el beneficio total de cada grupo de edad (que se obtiene multiplicando el monto de ganancia per cápita por el tamaño de la población con esas características). Posteriormente se reportan esos beneficios sociales totales como porcentaje del PIB. 
Los beneficios obtenidos son significativamente grandes dado que, aunque las ganancias per cápita sean moderadas, el tamaño de la población beneficiada produce beneficios sociales considerables. Es por ello que se dice que se presenta un efecto "masa" que amplifica los impactos de los programas de salud. En resumen, se puede mencionar que la erradicación de los infartos agudos de miocardio generan un beneficio económico social de $10.62 \%$ del PIB; la erradicación de la insuficiencia renal crónica un $2.87 \%$; la de los accidentes vasculares encefálicos agudos de $4.63 \%$; la de la neumonía de $4.79 \%$; la de las enfermedades pulmonares obstructivas crónicas de un 3.4\%; y la de los accidentes de tránsito de $0.43 \%$.

Es importante notar que estos resultados son mayores a los encontrados por Cerda y Torche (2005) para las enfermedades coincidentes en ambos estudios, ya que mientras para Chile la erradicación de la neumonía y los accidentes vasculares encefálicos agudos genera un beneficio social de alrededor de $2.89 \%$ y $1.48 \%$ del PIB respectivamente, en Nicaragua genera un $4.79 \%$ y $4.65 \%$ respectivamente. Esto se debe a que en Chile las tasas de mortalidad provocadas por estas enfermedades son mucho menores a las que dichas enfermedades generan en Nicaragua.

\section{Conclusiones}

El modelo de Cerda y Torche (2005), calibrado con información de la economía nicaragüense, es aplicable para la evaluación de políticas públicas en el sector salud. Esto fue demostrado con la evaluación de una política pública de erradicación de las seis principales causas de muertes en 2008 (infarto agudo de miocardio, insuficiencia renal crónica, accidentes vasculares encefálicos agudos, neumonía, enfermedad pulmonar obstructiva crónica y accidentes de tránsito).

Además, el modelo permitió realizar las primeras estimaciones del valor económico de la vida para Nicaragua. Para ello se siguió un enfoque teórico que muestra que este valor, a pesar de ser considerablemente alto, es muy inferior al encontrado en otros países como Chile (Cerda $\&$ Torche, 2005) y Estados Unidos (Murphy \& Topel, 1999). Sin embargo, es importante aclarar que dicho valor cambia considerablemente a través del ciclo de vida, así como entre individuos con diferente sexo y niveles educativos.

Así mismo, con los resultados del ejercicio práctico de la determinación del beneficio económico social de una política pública de erradicación de enfermedades específicas, se concluyó que un programa de erradicación de los infartos agudos de miocardio generaría un mayor beneficio económico social.

Finalmente, estos beneficios generados por la aplicación de programas de salud se pueden comparar con beneficios sociales generados por inversiones en otros sectores - como educación, demografía, pobreza y desigualdad -, de tal manera que se inviertan los recursos en el sector en donde generen un mayor rendimiento social por unidad monetaria gastada, permitiendo elevar el bienestar y desarrollo económico de la población nicaragüense. 


\section{Anexos}

Cuadro 4. Tabla de vida anual

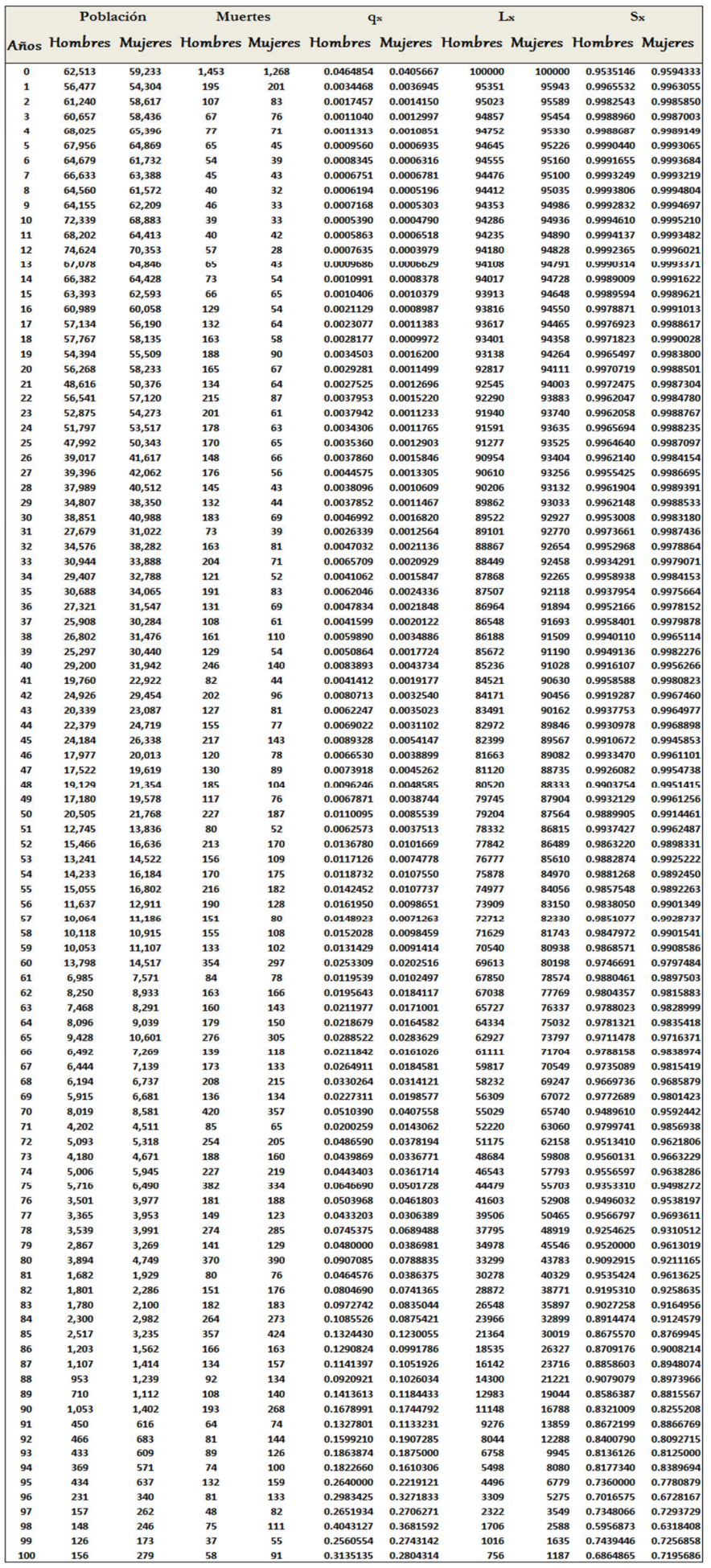


Encuentro №. 94, 7-41, 2013

Cuadro 5. Tabla de vida erradicando infarto agudo de miocardio

\begin{tabular}{|c|c|c|}
\hline & Pobla & ción \\
\hline Años & Hombres & Mujeres \\
\hline 0 & 62,513 & 59,233 \\
\hline 1 & 56,477 & 54,304 \\
\hline 2 & 61,240 & 58,617 \\
\hline 3 & 60,657 & 58,436 \\
\hline 4 & 68,025 & 65,396 \\
\hline 5 & 67,956 & 64,869 \\
\hline${ }_{7}^{6}$ & 64,679 & $\begin{array}{l}61,732 \\
63202\end{array}$ \\
\hline 8 & $\begin{array}{l}66,633 \\
664560\end{array}$ & $\begin{array}{l}63,388 \\
6,572\end{array}$ \\
\hline 9 & 64,155 & 62,209 \\
\hline 10 & 72,339 & 68,883 \\
\hline 11 & 68,202 & 64,413 \\
\hline 12 & 74,624 & 70,353 \\
\hline & & \\
\hline 14 & 66,382 & 64,428 \\
\hline 15 & 63,393 & 62,593 \\
\hline 16 & 60,989 & 60 \\
\hline 17 & 57,134 & 56,190 \\
\hline 18 & 57,767 & 58,135 \\
\hline 19 & 54,394 & 55,509 \\
\hline 20 & 56,268 & 58,233 \\
\hline 21 & 48,616 & 50,376 \\
\hline 22 & 56,544 & 57,122 \\
\hline 23 & 52,875 & 54,273 \\
\hline 24 & 51,797 & 53,517 \\
\hline 25 & $\begin{array}{l}47,992 \\
40027\end{array}$ & 50,343 \\
\hline $\begin{array}{l}26 \\
27 \\
27\end{array}$ & $\begin{array}{l}39,017 \\
39396\end{array}$ & $\begin{array}{l}41,617 \\
42,062\end{array}$ \\
\hline 28 & $\begin{array}{l}39,396 \\
37,989\end{array}$ & $\begin{array}{l}\begin{array}{l}42,062 \\
40,512\end{array} \\
4\end{array}$ \\
\hline 29 & 34,807 & 38,350 \\
\hline 30 & 38,851 & A0,088 \\
\hline 31 & 27,679 & 31,022 \\
\hline 32 & 34,576 & 38,282 \\
\hline 33 & 30,944 & 33,888 \\
\hline 34 & 29,407 & $\begin{array}{l}32,780 \\
32,788\end{array}$ \\
\hline 35 & 30,688 & 34,065 \\
\hline 36 & 27,321 & 31,547 \\
\hline 37 & 25,908 & 30,284 \\
\hline 38 & 26,802 & 31,476 \\
\hline 39 & 25,297 & 30,440 \\
\hline 40 & 29,200 & 31,942 \\
\hline 41 & 19,760 & 22,922 \\
\hline 42 & 24,926 & 29,454 \\
\hline 43 & 20,339 & 23,087 \\
\hline 44 & 22,379 & $\begin{array}{l}24,719 \\
2,392\end{array}$ \\
\hline $\begin{array}{l}45 \\
46 \\
46\end{array}$ & $\begin{array}{l}24,1844 \\
17997\end{array}$ & $\begin{array}{l}26,338 \\
20,013\end{array}$ \\
\hline 47 & 17,522 & $\begin{array}{l}2,015 \\
19,619\end{array}$ \\
\hline 48 & 19 & 21,354 \\
\hline 49 & 17,180 & 19,578 \\
\hline 50 & 20,505 & 21,768 \\
\hline 51 & 12,745 & 13,836 \\
\hline 52 & 15,466 & 16,636 \\
\hline 53 & 13,241 & 14,522 \\
\hline 54 & $\begin{array}{l}14,233 \\
1,055\end{array}$ & 16,184 \\
\hline 55 & $\begin{array}{l}15,055 \\
11,67\end{array}$ & $\begin{array}{l}16,802 \\
12012\end{array}$ \\
\hline $\begin{array}{l}56 \\
57 \\
57\end{array}$ & $\begin{array}{l}11,637 \\
10,064\end{array}$ & $\begin{array}{l}\begin{array}{l}12,911 \\
111,186\end{array} \\
1\end{array}$ \\
\hline 58 & $\begin{array}{l}10,0,04 \\
10,118\end{array}$ & $\begin{array}{l}11,1186 \\
10,915\end{array}$ \\
\hline 59 & 10,053 & 11,107 \\
\hline 60 & 13,798 & 14,517 \\
\hline 61 & 6,985 & \\
\hline 62 & 8,250 & 8,933 \\
\hline 63 & 7,468 & 8,291 \\
\hline 64 & 8,096 & 9,039 \\
\hline 65 & 0,128 & 10,601 \\
\hline 66 & 6,492 & 7,269 \\
\hline 67 & 6,424 & 7,139 \\
\hline 68 & 6,194 & 6,737 \\
\hline 69 & 5,915 & 6,681 \\
\hline 70 & 8,019 & 8,581 \\
\hline 71 & 4,202 & 4,511 \\
\hline 72 & 5,093 & 5,318 \\
\hline 73 & 4,180 & 4,671 \\
\hline${ }_{75}^{14}$ & $\begin{array}{l}5,000 \\
5,716\end{array}$ & $\begin{array}{l}5,945 \\
6,990\end{array}$ \\
\hline 76 & $\begin{array}{l}3,501 \\
3,501\end{array}$ & $\begin{array}{l}0,490 \\
3,977\end{array}$ \\
\hline 77 & 3,3 & 3,9 \\
\hline 78 & 3,5 & 3,9 \\
\hline 79 & 2,867 & 3,2 \\
\hline 80 & & 4,7 \\
\hline 81 & 1,6 & 1,9 \\
\hline 82 & 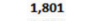 & 2,2 \\
\hline 83 & 1,780 & 2,100 \\
\hline 84 & 2,300 & 2,982 \\
\hline 85 & 2,5 & 3,235 \\
\hline 86 & 1,203 & 1,562 \\
\hline 87 & $\begin{array}{l}1,107 \\
953\end{array}$ & 1,414 \\
\hline 89 & $\begin{array}{l}953 \\
710\end{array}$ & \\
\hline & 10 & \\
\hline 91 & & 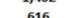 \\
\hline . & 466 & $\begin{array}{l}61 \\
68\end{array}$ \\
\hline 93 & 43 & $\begin{array}{l}689 \\
609\end{array}$ \\
\hline 94 & 369 & 57 \\
\hline 95 & 434 & 637 \\
\hline 96 & 231 & 340 \\
\hline 97 & 157 & 262 \\
\hline 98 & 148 & 20 \\
\hline 99 & 126 & 173 \\
\hline
\end{tabular}


Cuadro 6. Tabla de vida erradicando insuficiencia renal crónica

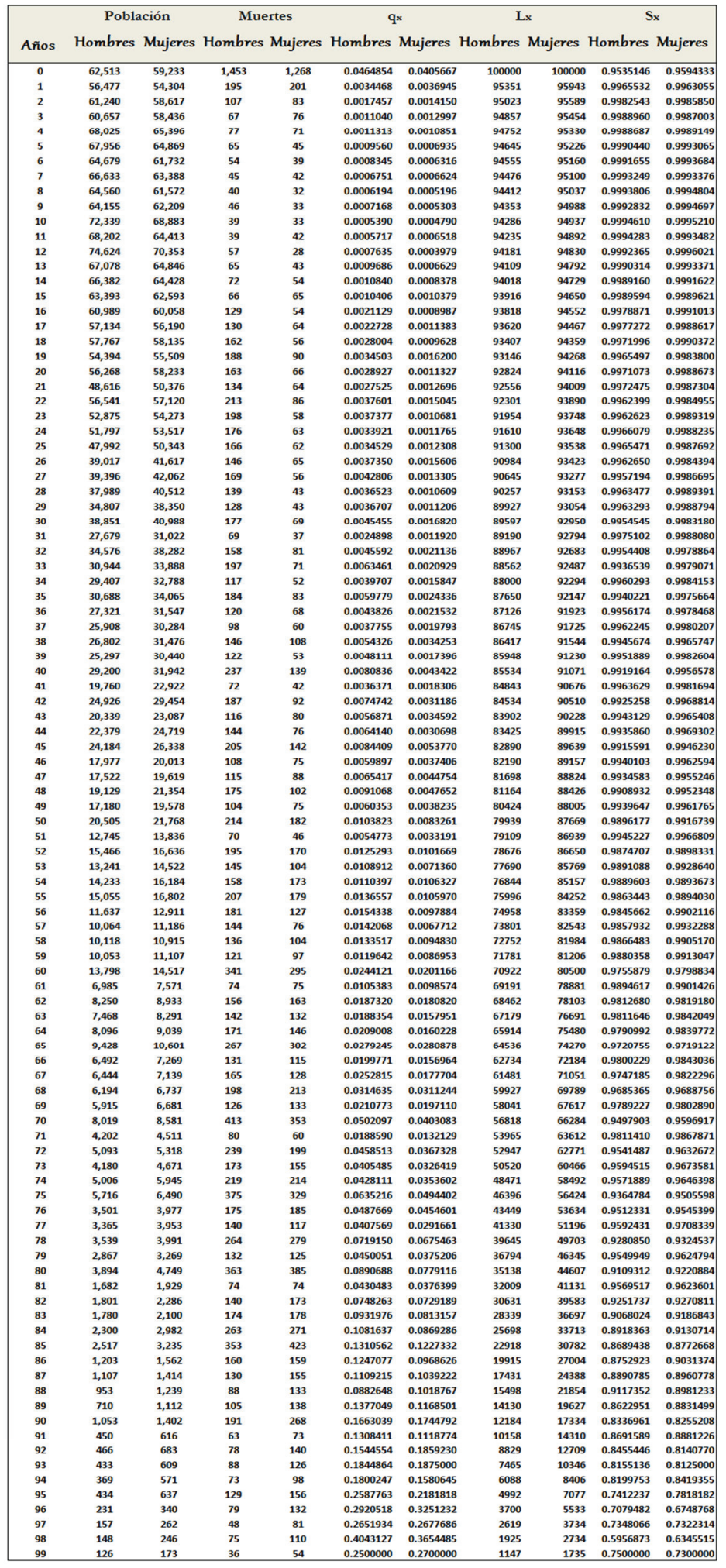


Encuentro No. 94, 7-41, 2013

Cuadro 7. Tabla de vida erradicando accidente vascular encefálico agudo

\begin{tabular}{|c|}
\hline \\
\hline Años \\
\hline 0 \\
\hline 1 \\
\hline 2 \\
\hline 3 \\
\hline 4 \\
\hline 5 \\
\hline 6 \\
\hline 7 \\
\hline 8 \\
\hline 9 \\
\hline 10 \\
\hline 11 \\
\hline 12 \\
\hline \\
\hline 14 \\
\hline 15 \\
\hline 16 \\
\hline 17 \\
\hline 18 \\
\hline 19 \\
\hline 20 \\
\hline 21 \\
\hline 22 \\
\hline 23 \\
\hline 24 \\
\hline 25 \\
\hline $\begin{array}{l}26 \\
27\end{array}$ \\
\hline $\begin{array}{l}27 \\
28\end{array}$ \\
\hline 29 \\
\hline 30 \\
\hline 31 \\
\hline 32 \\
\hline 33 \\
\hline 34 \\
\hline 35 \\
\hline 36 \\
\hline 37 \\
\hline 38 \\
\hline 39 \\
\hline 40 \\
\hline 41 \\
\hline 42 \\
\hline 43 \\
\hline 44 \\
\hline 45 \\
\hline 46 \\
\hline 47 \\
\hline 48 \\
\hline $\begin{array}{l}49 \\
5\end{array}$ \\
\hline $\begin{array}{l}50 \\
51\end{array}$ \\
\hline $\begin{array}{l}51 \\
52\end{array}$ \\
\hline 53 \\
\hline 54 \\
\hline 55 \\
\hline \\
\hline 57 \\
\hline 58 \\
\hline 59 \\
\hline 60 \\
\hline 61 \\
\hline 62 \\
\hline 63 \\
\hline 64 \\
\hline 65 \\
\hline 66 \\
\hline 67 \\
\hline 68 \\
\hline 69 \\
\hline 70 \\
\hline 71 \\
\hline 72 \\
\hline 73 \\
\hline $\begin{array}{l}74 \\
775\end{array}$ \\
\hline $\begin{array}{l}75 \\
76\end{array}$ \\
\hline \\
\hline 78 \\
\hline 79 \\
\hline 80 \\
\hline 81 \\
\hline 82 \\
\hline 83 \\
\hline 84 \\
\hline 85 \\
\hline 86 \\
\hline $\begin{array}{l}87 \\
88\end{array}$ \\
\hline 88 \\
\hline \\
\hline 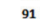 \\
\hline 92 \\
\hline 93 \\
\hline 94 \\
\hline 95 \\
\hline 96 \\
\hline 97 \\
\hline 98 \\
\hline 99 \\
\hline
\end{tabular}


Cuadro 8. Tabla de vida erradicando neumonía

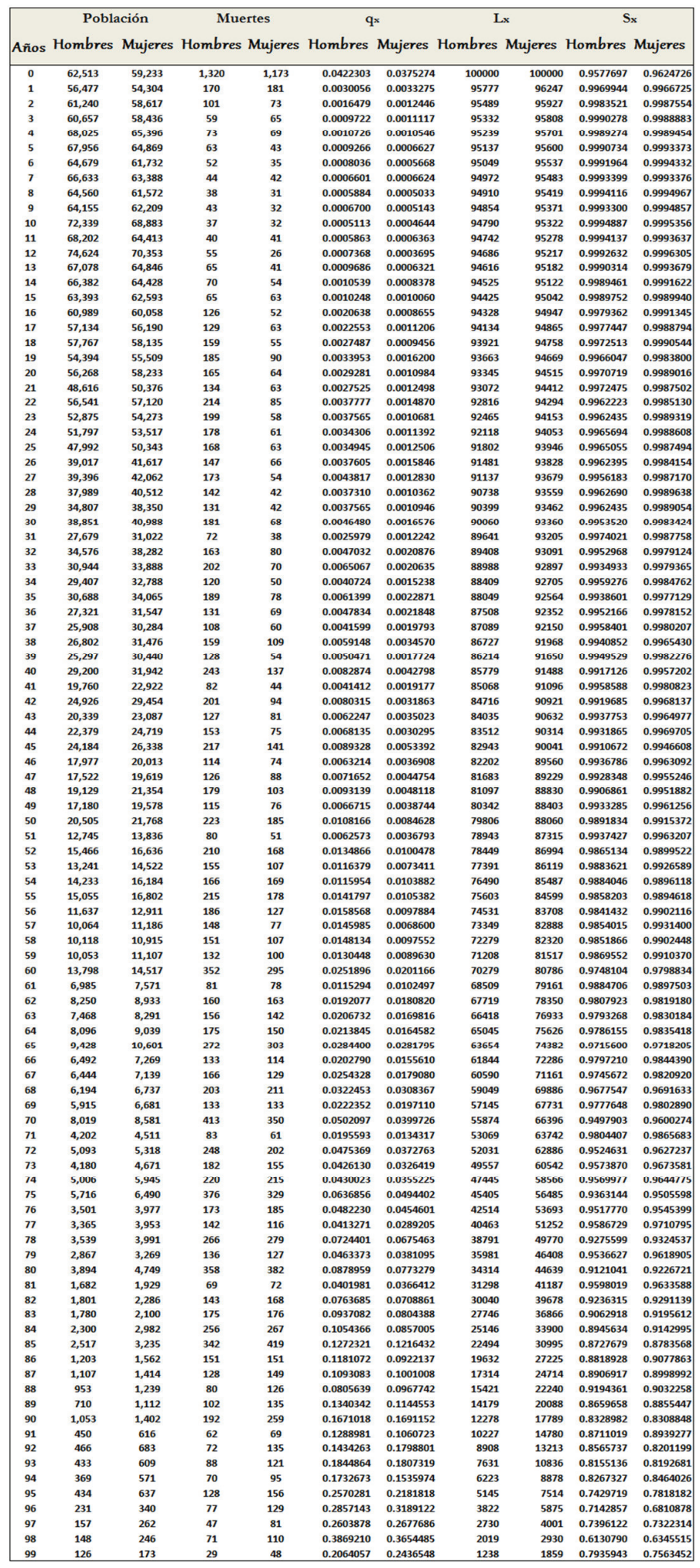


Encuentro №. 94, 7-41, 2013

Cuadro 9. Tabla de vida erradicando enfermedad pulmonar obstructiva crónica

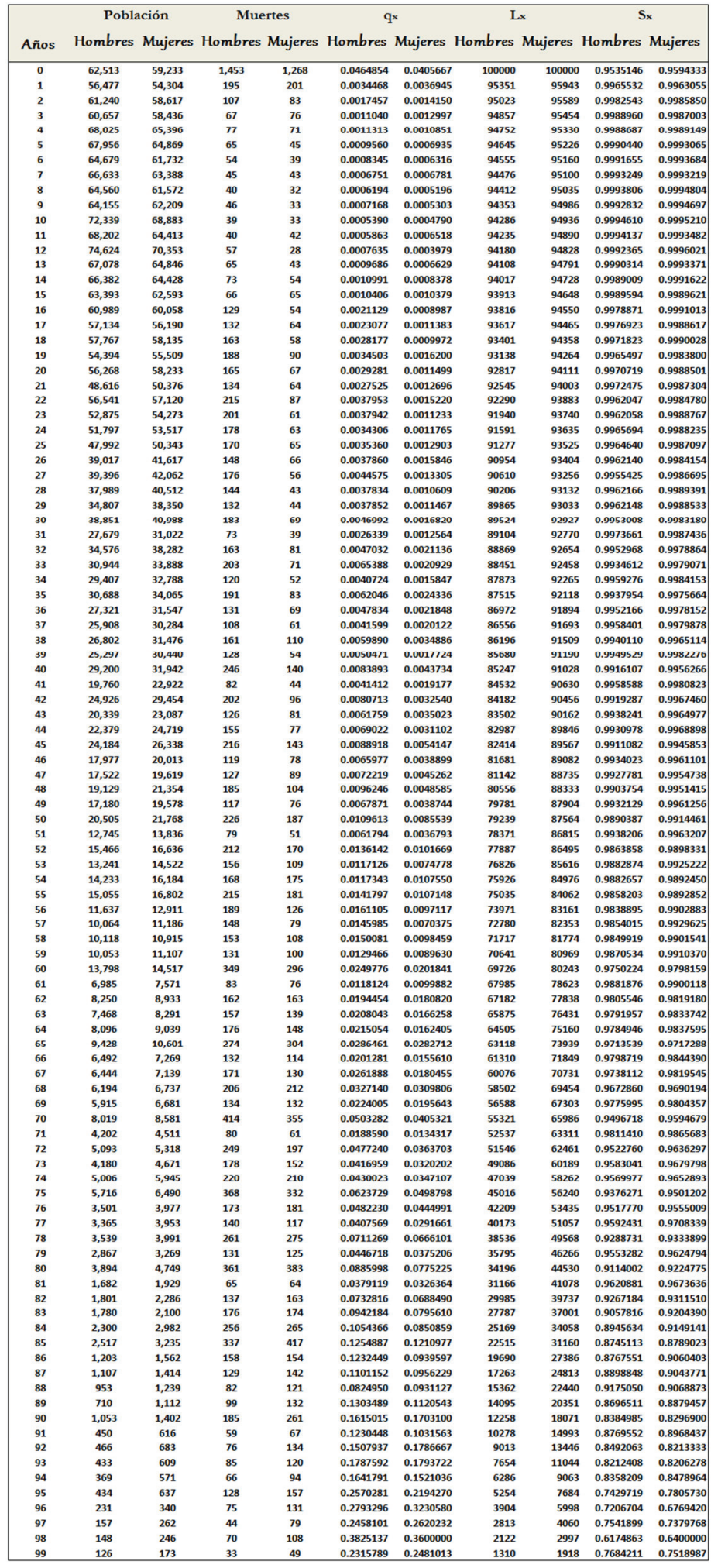


Cuadro 10. Tabla de vida erradicando accidentes de tránsito

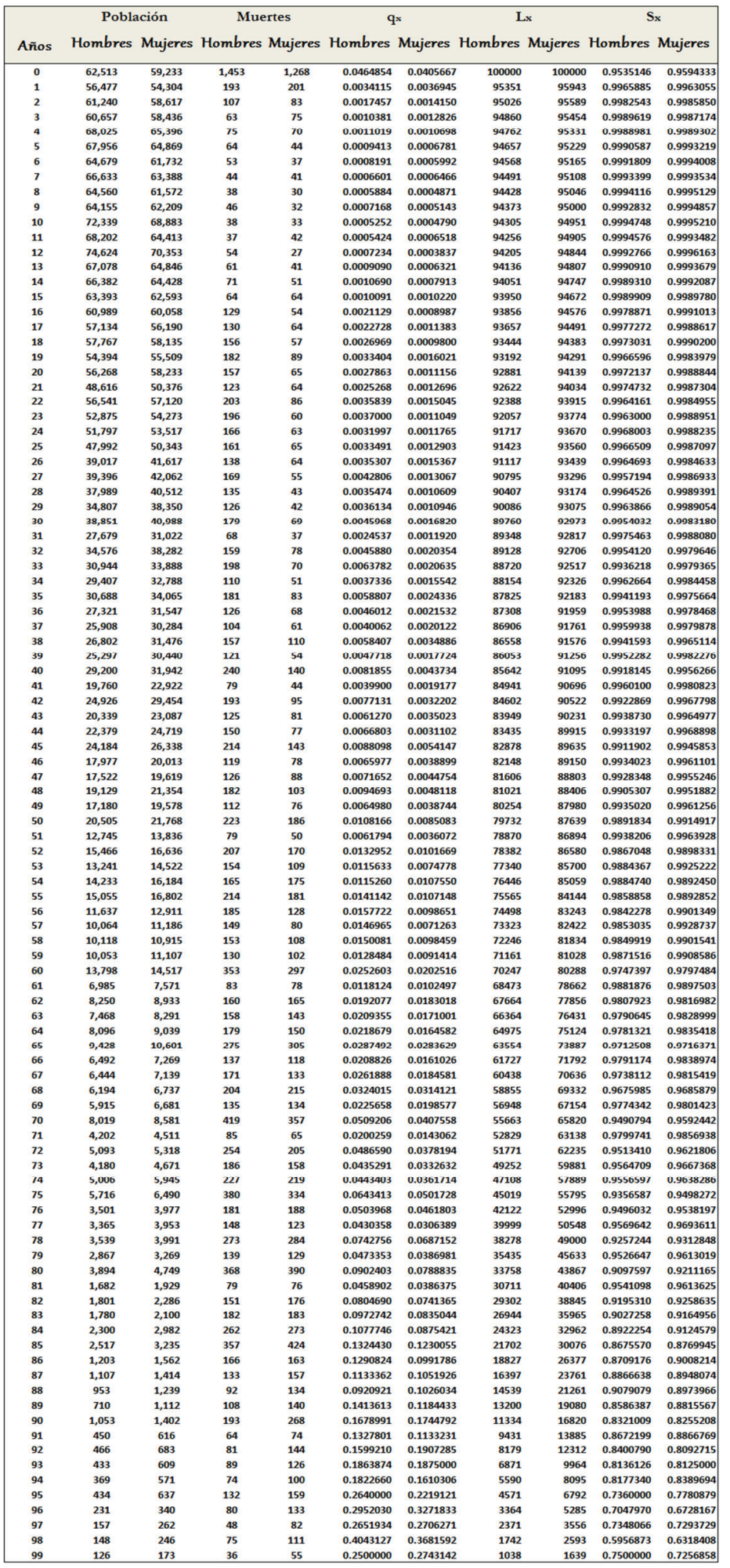


Encuentro №. 94, 7-41, 2013

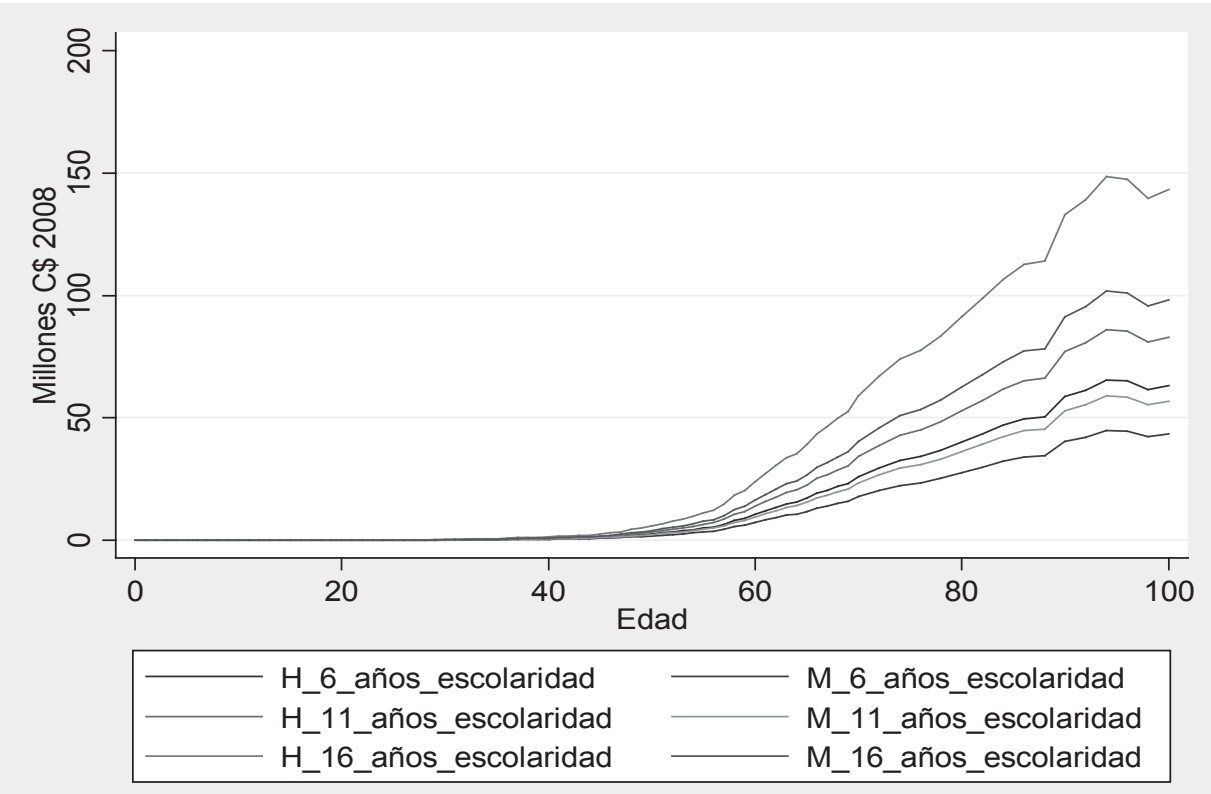

Ilustración 5. Valor de eliminar muertes por infarto agudo de miocardio

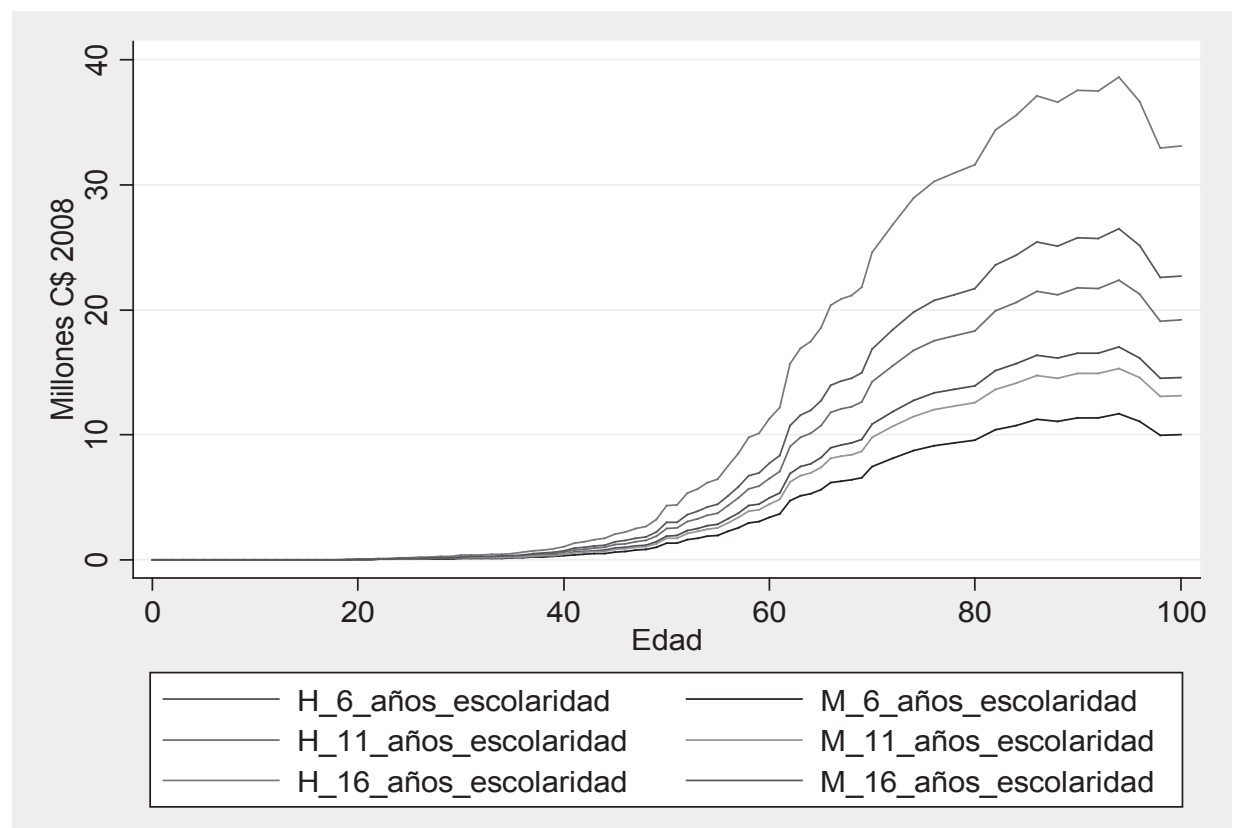

Ilustración 6. Valor de eliminar muertes por insuficiencia renal crónica 


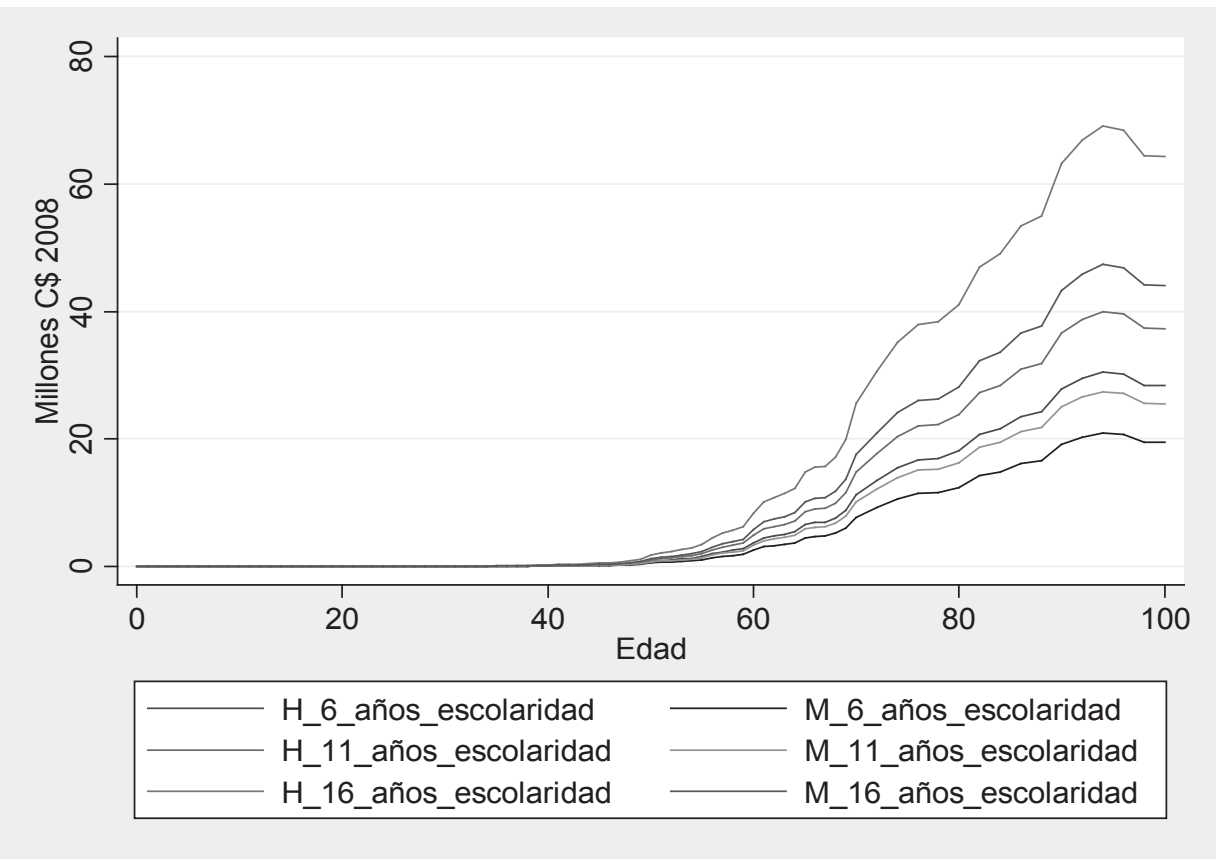

Ilustración 7. Valor de eliminar muertes por accidente vascular encefálico agudo

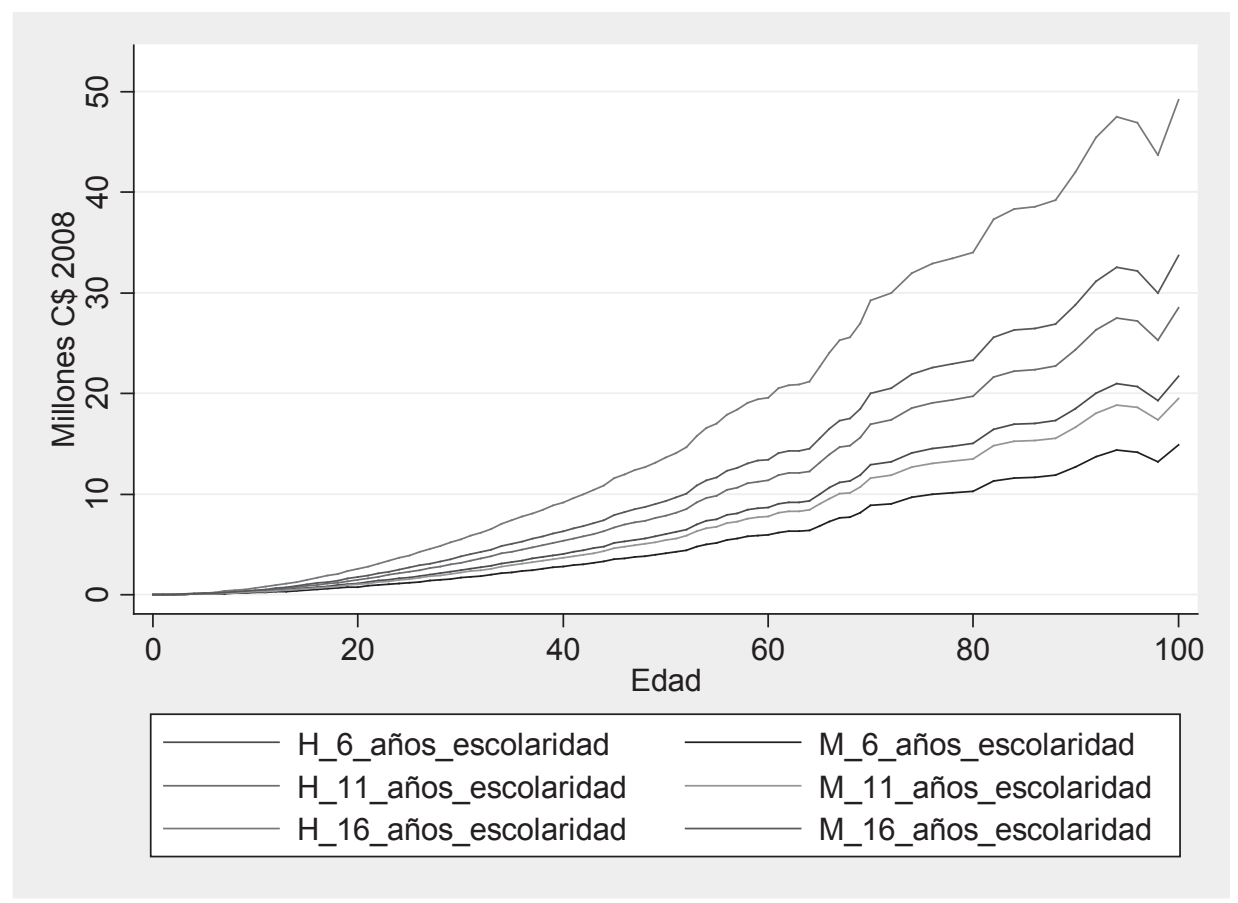

Ilustración 8. Valor de eliminar muertes por enfermedad pulmonar obstructiva crónica 
Encuentro №. 94, 7-41, 2013

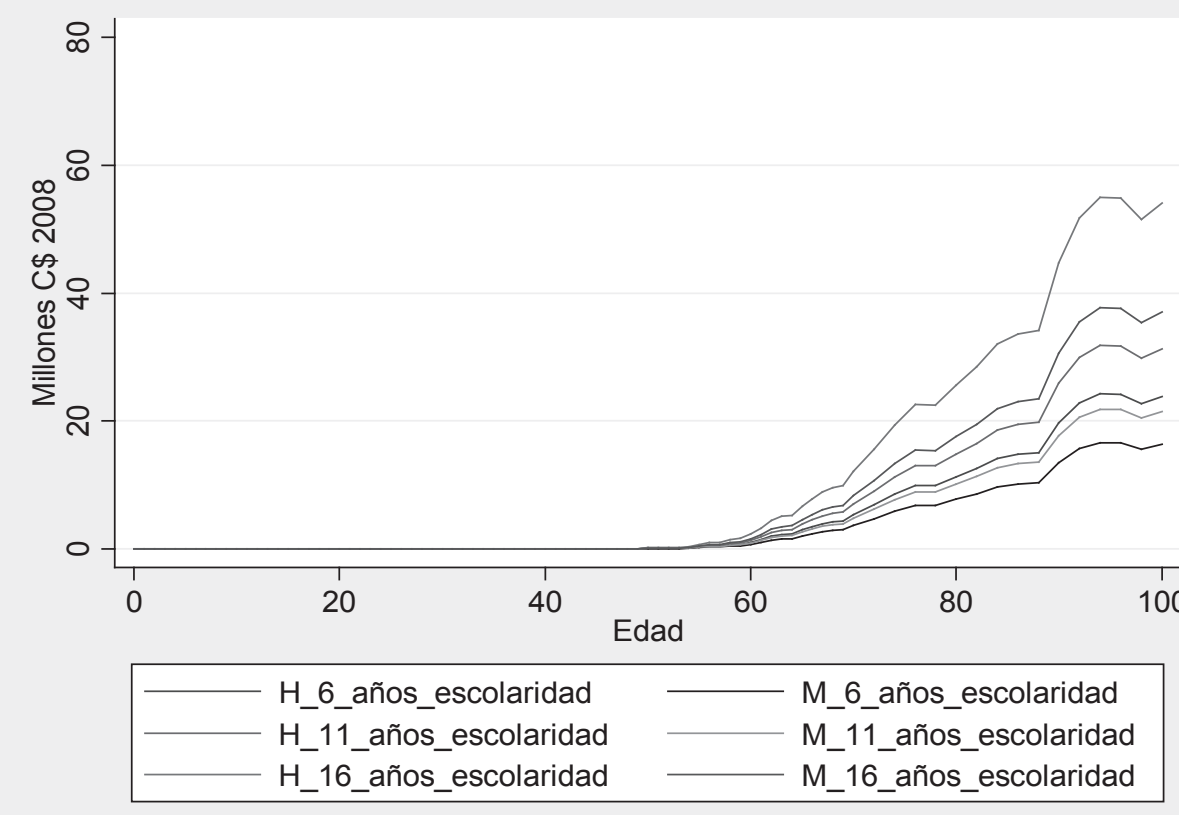

Ilustración 9. Valor de eliminar muertes por enfermedad pulmonar obstructiva crónica

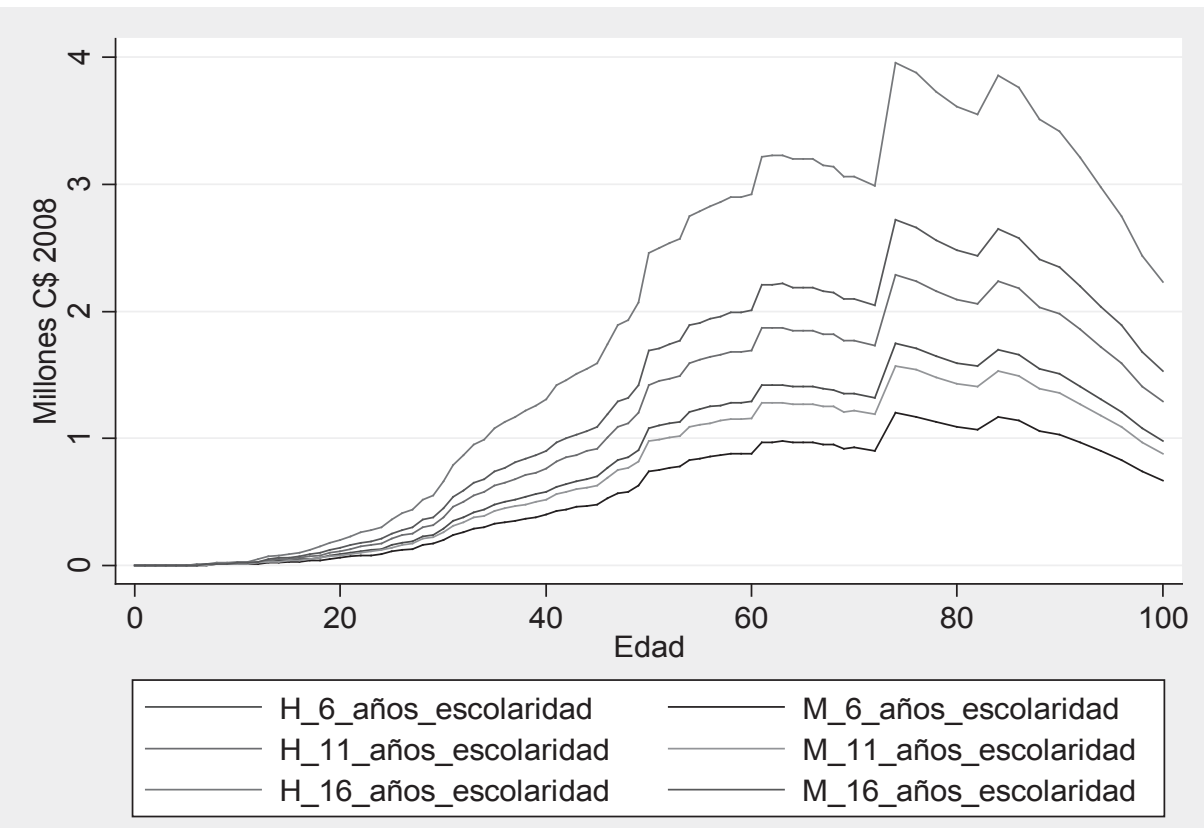

Ilustración 10. Valor de eliminar muertes por accidentes de tránsito 
Cuadro 11. Valor económico de erradicar infarto agudo de miocardio, insuficiencia renal crónica y accidente vascular encefálico agudo. Miles de millones C\$2008.

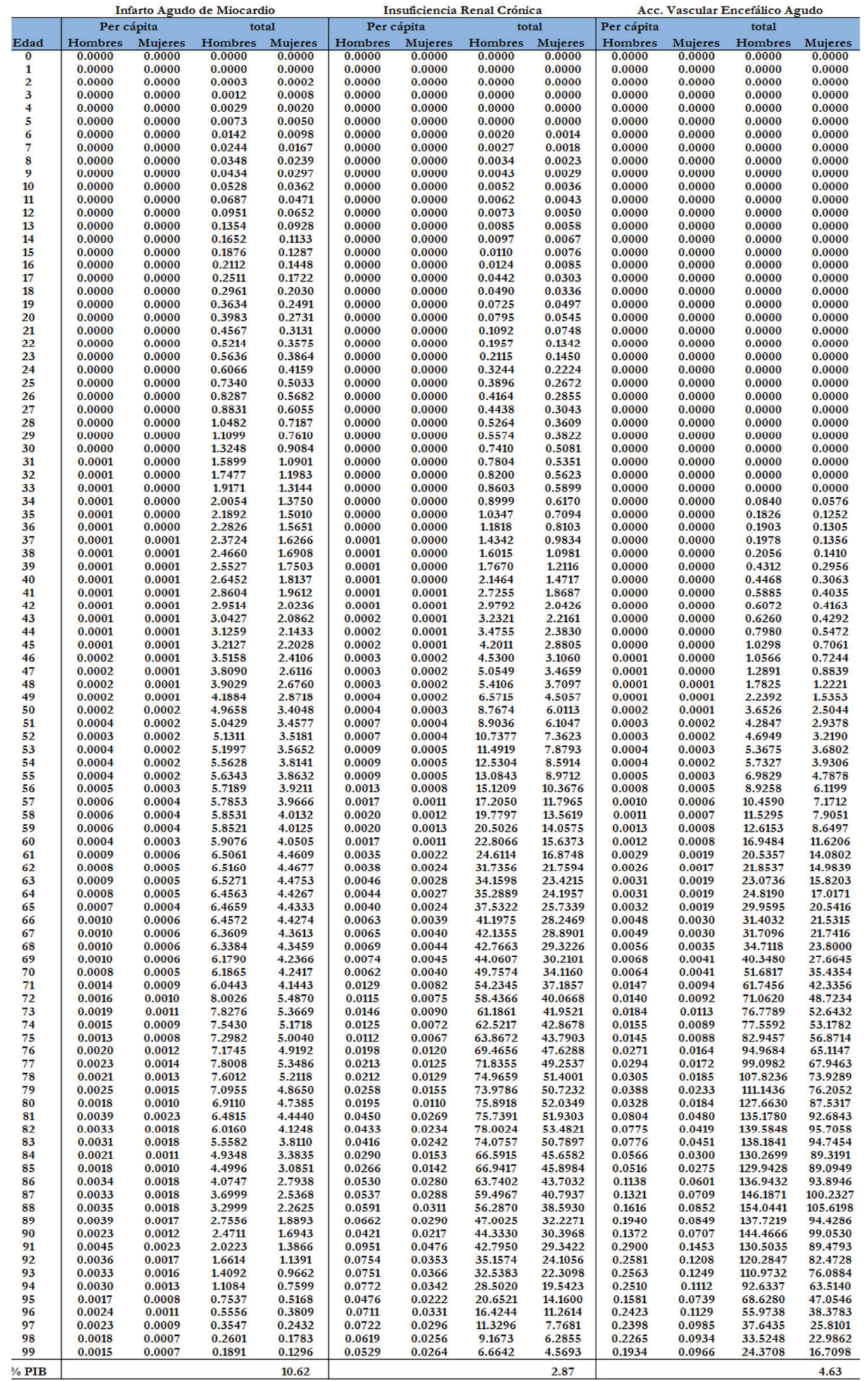


Cuadro 12. Valor económico de erradicar neumonía, enfermedad pulmonar obstructiva crónica y accidentes de tránsito. Miles de millones C\$2008.

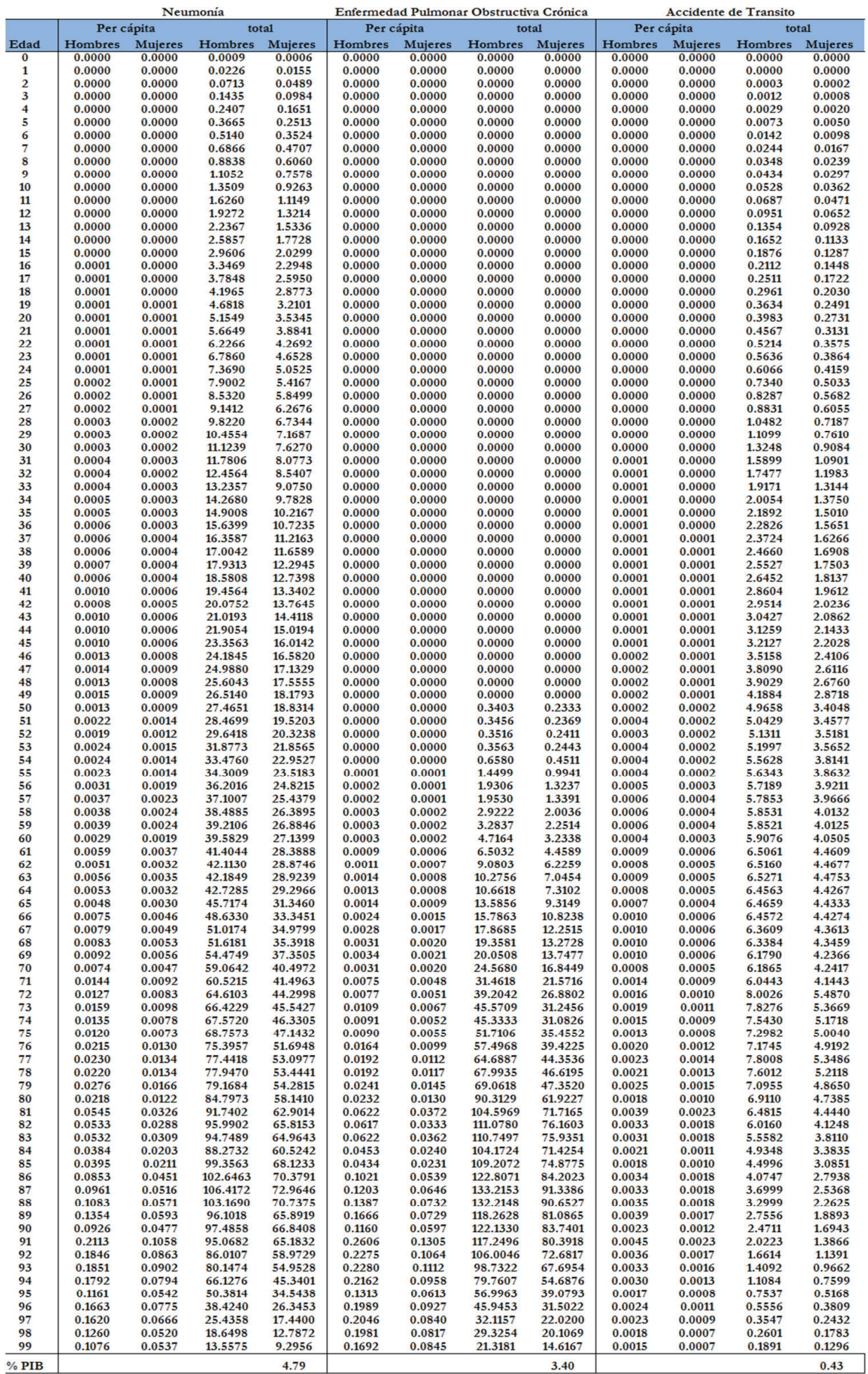




\section{Referencias bibliográficas}

Arthur, W. (1981). The Economics of Risks to Life. A.E.R. (71), 54-64.

Cerda, R. \& Torche, A. (2005). El valor económico de reducir tasas de mortalidad: El caso de Chile. Documento de trabajo No. 285, Santiago: Pontificia Universidad Católica de Chile, Departamento de Economía.

Conferencia Interamericana de Seguridad Social. [CISS]. (2009). Preferencias, Gasto en Salud y el Valor económico de la vida Estadistica en América. CISS Working Paper No. 2009/01, 2009b. Inter-American Conference on Social Security. Recuperado el 20 de enero de 2011, de www.ciss.org.mx/pdf/es/estudios/CISSWP-0901.pdf

Cropper, M. \& Freeman, A. (1991). Environmental Health Effects, en Braden, J. B. \& Kolstad, C. D. Contribution to Economic Analysis: Measuring the Demand for Environmental Quality. (pp. 117-140). Amsterdam: Elsevier Science Publications.

Cropper, M., Aydede, S. \& Portney P. (1992). Rates of Time Preference for Saving Lives. American Economic Review, 82 (2), 469-473.

Heckman, J. (1979). Sample selection bias as a specification error. Econometrica, 47 (1), 153-161.

Instituto Nacional de Información de Desarrollo [INIDE]. (2009). Encuesta de Medición del Empleo-EME. Soporte en CD. Managua: Autor.

Jones-Lee, M., Hammerton, M. \& Phillips, P. (1985). The Value of Safety: Results of a National Sample Survey. The Economic Journal, (95), 49-72.

Marin, A. \& Psacharopoulos, G. (1982). The Reward for Risk in the Labor Market: Evidence from the United Kingdom and a Reconciliation with Other Studies. Journal of Economical Economy, 90 (4), 827-853.

Martínez, J., Abellán, J. \& Pinto, J. (2007). El valor monetario de la vida estadística en España a través de las preferencias declaradas. Instituto de Estudios Fiscales, Hacienda Pública Española. Revista de Economía Pública, 183 (4), 125-144.

Mincer, J. (1974). Schooling, Experience, and Earnings. New York: NBER Press.

Murphy, K. \& Topel, R. (1999). The economic value of medical research. Chicago: University of Chicago Business School.

Murphy, K. \& Topel, R. (2006). The Value of Health and Longevity. Journal of Political Economy, 114 (5), 871-904.

National Center for Health Statistics. (1999). Method for Constructing Complete Annual U.S. Life Tables. Recuperado el 07 de febrero de 2011, de http://www.cdc. gov/nchs/products/life_tables.htm

Riera, A., Ripoll, A. \& Mateu, J. (2007). Estimación del valor estadístico de la vida en España: Una aplicación del método de salarios hedónicos. Instituto de Estudios Fiscales, Hacienda Pública Española. Revista de Economía Pública, 181 (4), 29-48.

Rosen, S. (1988). The Value of Changes in Life Expectancy. Journal of Risk and Uncertainty J. Rish and Uncertainly, 1(3), 285-304.

Viscusi, W. (1993). The Value of Risks to Life and Health. Journal of Economic Literature, 31 (4), 1912-1946.

Viscusi, W. \& Moore, M. (1989). Rates of Time Preference and Valuation of the Duration of Life. Journal of Public Economics, (38), 297-31. 